\title{
Positive emotion broadens attention focus through decreased position-specific spatial encoding in early visual cortex: Evidence from ERPs
}

\author{
Naomi Vanlessen • Valentina Rossi • Rudi De Raedt • Gilles Pourtois
}

Published online: 23 October 2012

(C) Psychonomic Society, Inc. 2012

\begin{abstract}
Recent evidence has suggested that not only stimulus-specific attributes or top-down expectations can modulate attention selection processes, but also the actual mood state of the participant. In this study, we tested the prediction that the induction of positive mood can dynamically influence attention allocation and, in turn, modulate early stimulus sensory processing in primary visual cortex (V1). High-density visual event-related potentials (ERPs) were recorded while participants performed a demanding task at fixation and were presented with peripheral irrelevant visual textures, whose position was systematically varied in the upper visual field (close, medium, or far relative to fixation). Either a neutral or a positive mood was reliably induced and maintained throughout the experimental session. The ERP results showed that the earliest retinotopic component following stimulus onset (C1) strongly varied in topography as a function of the position of the peripheral distractor, in agreement with a near-far spatial gradient. However, this effect was altered for participants in a positive relative to a neutral mood. On the contrary, positive mood did not modulate attention allocation for the central (taskrelevant) stimuli, as reflected by the P300 component. We ran a control behavioral experiment confirming that positive emotion selectively impaired attention allocation to the peripheral distractors. These results suggest a mood-dependent tuning of position-specific encoding in V1 rapidly following stimulus onset. We discuss these results against the dominant broaden-and-build theory.
\end{abstract}

N. Vanlessen $\cdot$ V. Rossi $\cdot$ R. De Raedt $\cdot$ G. Pourtois $(\bowtie)$

Psychopathology and Affective Neuroscience Laboratory,

Department of Experimental-Clinical and Health Psychology,

Ghent University,

Henri Dunantlaan 2,

9000 Gent, Belgium

e-mail: gilles.pourtois@ugent.be
Keywords Positive emotion - ERP · C1 · Attention . Inhibition $\cdot$ Selectivity $\cdot$ Mood induction

Sensory stimulus processing is determined not only by bottom-up physical characteristics, but also by top-down cognitive or affective processes. In this framework, mood has been shown to shape the way that incoming information is attended and eventually processed (Gray, 2001, 2004). According to Fredrickson's influential broaden-and-build theory, negative and positive emotions have opposing but complementary functions (Fredrickson \& Levenson, 1998): While negative emotions can narrow the thought-action repertoires of an individual, positive affect can substantially broaden thinking styles and these thought-action repertoires. Positive affective states elicit a broadening of the scope of attention (Derryberry \& Reed, 1994), eventually enabling an open (Estrada, Isen, \& Young, 1997), creative (Isen, Daubman, \& Nowicki, 1987), integrative (Isen, Rosenzweig, \& Young, 1991), and flexible (Isen \& Daubman, 1984) way of thinking. In a similar vein, cognitive control abilities, and more specifically conflict adaptation, are reduced following the transient induction of positive mood (van Steenbergen, Band, \& Hommel, 2009, 2010).

Interestingly, recent findings have suggested that a weakening of inhibitory control processes provides a plausible mechanism to account for a broadening of attention after the induction of positive emotion (Rowe, Hirsh, \& Anderson, 2007; Wang et al., 2011). In this framework, loosening up inhibitory processes would result in a broader informationprocessing style, and hence a less narrowed attention focus. As a result, individuals in a happy mood would become more receptive to irrelevant information, allowing distracting stimuli to interfere more strongly with goal-relevant stimuli (Dreisbach \& Goschke, 2004). Thus, positive 
emotion would primarily reduce inhibitory control mechanisms (i.e., decrease attention selectivity), eventually leading to a less selective mode of stimulus processing, consistent with the predictions of the broaden-and-build theory. While this mechanism could, on the one hand, explain enhanced distractibility under positive mood, it might, on the other hand, also enable people to think in a more creative and flexible way, because they could learn more efficiently from incidental opportunities (Biss \& Hasher, 2011; Biss, Hasher, \& Thomas, 2010). Whereas many studies have already focused on these gains and drawbacks in higher-level cognition and reasoning under positive mood (Ashby, Isen, \& Turken, 1999; Biss \& Hasher, 2011; Biss et al., 2010; Fredrickson, 2001; Fredrickson \& Levenson, 1998; Isen, 2000), the actual modus operandi of positive emotion on attention abilities still remains largely underspecified. More specifically, an unanswered question is how positive emotion may dynamically shape and transform attention control mechanisms, such that a broader attentional scope can eventually bias early sensory stimulus processing, leading to the enhancement of both creativity and distractibility. Decreased attention control in this condition might underlie these behavioral phenomena.

Attention control usually refers to the different brain mechanisms that enable fast and efficient selection of relevant information in the environment (Desimone \& Duncan, 1995; Posner, Snyder, \& Davidson, 1980). Not only do perceptual salience, novelty, and unexpectedness determine the ease of attentional selection (bottom-up factors; see Egeth \& Yantis, 1997; Wolfe \& Horowitz, 2004), but prior knowledge, expectations, and mood reliably influence early sensory stimulus processing (top-down factors; see Corbetta \& Shulman, 2002). Thus, both top-down and bottom-up attention processes exert control over sensory stimulus processing in such a way as to gate the flow of incoming information and eventually to facilitate the selection of relevant stimuli, while filtering irrelevant information from further processing (Corbetta \& Shulman, 2002; Desimone \& Duncan, 1995; Theeuwes, 2010). Interestingly, it has been suggested that prefrontal cognitive control regions are swiftly recruited in order to downplay the interfering effect potentially created by distractors, and eventually to maintain an efficient attention selection process (Lavie, 2005; Lavie, Hirst, de Fockert, \& Viding, 2004). However, these attention control systems are dynamic and not immune to changes in affective states (see Desseilles et al., 2009; Gray, 2004; Rossi \& Pourtois, 2012; Rowe et al., 2007). Nonetheless, the effects of positive emotion on these attention control mechanisms, which gate sensory processing as early as in V1, have been much less explored than the effects of negative emotion (Stolarova, Keil, \& Moratti, 2006; West, Anderson, Ferber, \& Pratt, 2011).
Accordingly, the goal of our study was to investigate, using state-of-the-art event-related potential (ERP) methods (Exp. 1) and standard behavioral measures (Exp. 2), possible downside effects of positive mood on early sensory stimulus processing, presumably resulting from a change in top-down attention control mechanisms. Participants performed a demanding task at fixation, while distractors were presented in the upper visual field at unpredictable times and locations relative to the task-relevant stimuli (Schwartz et al., 2005). This setup is suited to explore, using high-density electroencephalography (EEG) measurements, changes in the spatial gradient of visual attention toward peripheral distractors, while fixation is maintained at a constant location in the center of the screen (Pourtois, Delplanque, Michel, \& Vuilleumier, 2008; Rauss, Schwartz, \& Pourtois, 2011; Rossi \& Pourtois, 2012). Because we used eccentric/peripheral visual stimuli, we could record a reliable C1 ERP component that reflects early retinotopic encoding of the stimulus in V1 and yet is sensitive to topdown attention control effects (Rauss, Pourtois, Vuilleumier, \& Schwartz, 2009; Rauss et al., 2011; Rossi \& Pourtois, 2012). The $\mathrm{C} 1$ usually peaks $\sim 50-100 \mathrm{~ms}$ after stimulus onset over central occipito-parietal scalp positions (Di Russo, Martínez, Sereno, Pitzalis, \& Hillyard, 2002; Jeffreys \& Axford, 1972). In accordance with the cruciform organization of the primary visual cortex and calcarine fissure, the amplitude and polarity of the $\mathrm{C} 1$ substantially change as a function of the position of the stimulus in the visual field (Clark, Fan, \& Hillyard, 1995).

In Experiment 1, we therefore capitalized on these welldefined electrophysiological properties to investigate whether the $\mathrm{C} 1$ component could vary in size and topography not only according to the actual position of a distractor stimulus shown in the upper visual field (i.e., a larger negative component for the position close to fixation, relative to those farther away), but also with the actual mood of the participant (either happy or neutral). We tested the prediction that the selectivity for early spatial encoding of distractors in V1, at the level of the $\mathrm{C} 1$, would decrease after the induction of positive mood. This effect could result from a modulation of top-down attention control mechanisms by positive mood (Rowe et al., 2007), resulting in decreased position-specific selectivity at a more basic perceptual level. More precisely, we surmised that the normal reduction of the $\mathrm{C} 1$ with increasing distance of the distractor, relative to fixation, would be less pronounced in participants in a positive mood, relative to a neutral mood, consistent with a broadening of attention.

We also explored the possible effects of positive mood on later ERP components in response to peripheral distractors. Unlike the striate $\mathrm{C} 1$, the extrastriate $\mathrm{P} 1$, which peaks $\sim 100$ $150 \mathrm{~ms}$ post-stimulus-onset over occipital leads, is mostly sensitive to the content and not to the position of the 
stimulus within the visual field (Gomez Gonzalez, Clark, Fan, Luck, \& Hillyard, 1994; Herrmann \& Knight, 2000; Martínez et al., 1999), but it can also vary depending on the affective state of the participant (Moriya \& Nittono, 2011). Moreover, the amplitude of the P1 is typically larger for attended, relative to unattended, stimuli, consistent with a gain control mechanism of visual attention exerting modulatory effects in the extrastriate visual cortex (Hillyard \& Anllo-Vento, 1998; Martínez et al., 1999). Finally, we were also interested in the possible effects of mood on the processing of central, task-relevant stimuli. The efficiency of central target stimulus processing was mostly assessed by means of the decision-related P300 component, whose amplitude varies strongly with the amount of resources allocated to task demands (Ericsson, Olofsson, Nordin, Rudolfsson, \& Sandstrom, 2008; Kok, 2001; Polich, 2007; Polich \& Kok, 1995). Moreover, because this component has recently been shown to vary with the (negative) affective state of the participant (e.g., Shackman, Maxwell, McMenamin, Greischar, \& Davidson, 2011), we tested whether or not attention allocation toward central (taskrelevant) stimuli could also be altered after the induction of positive mood.

To corroborate the assumption of a drop in early attention selectivity for peripheral textures following the induction of positive mood, we ran an additional behavioral experiment. In Experiment 2, participants were asked to explicitly discriminate the content of these peripheral stimuli (in addition to the centrally presented stimuli at fixation). We reasoned that if the effect of positive mood may correspond to a change in prefrontal attention control mechanisms, and hence to early sensory stimulus selectivity, the visual processing of these peripheral stimuli may be impaired as compared to a control condition (i.e., neutral mood).

Previous research has shown that increased attentional scope is associated with a loss in spatial resolution and processing efficiency, as compared to detailed processing when the attentional scope is narrowed to a specific portion of the visual field (Carrasco, 2011; Castiello \& Umiltà, 1990, 1992; Eriksen \& Yeh, 1985; Ivry \& Robertson, 1998; Müller, Bartelt, Donner, Villringer, \& Brandt, 2003). Thus, if positive mood can broaden the attentional scope, processing over larger portions of the space can cause a drop in spatial resolution, as compared to a smaller attentional scope in a neutral mood. Hence, such an impairment in early spatial-encoding selectivity would, in turn, constrain the capacity to perform a visual discrimination of peripheral stimuli on the basis of processing of local (geometric) features.

Experiment 2 also enabled us to confirm whether the processing of the centrally presented stimuli would be unchanged in a positive relative to a neutral mood, in line with the results obtained in Experiment 1.

\section{Method}

\section{Participants}

We tested 70 participants who were all right-handed, had normal or corrected-to-normal vision, and had no history of psychiatric or neurologic disorder according to a selfreport questionnaire. All of the participants were undergraduate psychology students from Ghent University, and all gave written informed consent prior to participation. Of the 34 participants recruited for Experiment 1 (age: $M=22, S D=2$; seven male, 27 female), 17 were randomly assigned to the positive mood condition and 17 to the neutral mood condition. One participant in the neutral mood group reported not being able to use imagery, making the implementation of the mood induction procedure (MIP) impossible. Therefore, the data for this participant were excluded from further analyses. In Experiment 2, 36 undergraduate students (age: $M=19$, $S D=1$; three male, 33 female) were randomly assigned to either the neutral or the positive mood condition (18 participants per condition). The data of one participant from the neutral mood group were excluded from further analysis because of excessively slow RTs for the detection of central targets (mean $\pm 2.5 S D s$ ). Results are reported for the 35 remaining participants. The students participated in exchange for either course credits (32) or financial compensation (38).

\section{Materials}

Mood induction We used a between-subjects design to induce either a sustained positive or neutral/control mood, a design suited to avoid possible carryover effects between different mood conditions. Participants were naïve regarding the purpose of the MIP. A cover story was told in order to make the participants believe that the experiment concerned the relationship between the processing of visual information and the use of imagination. Mood was induced by means of an imagery procedure, according to which the participants were instructed to vividly imagine reliving a happy or neutral autobiographical memory (Holmes, 2006; Holmes, Coughtrey, \& Connor, 2008). First, the participants were trained in taking a field perspective (i.e., imagining from one's own perspective) during mental imagery (Holmes, 2006; Holmes et al., 2008). Next, they were instructed to recall and report, using episodic memory, an event that happened on a specific day, more than one week previously, that either made them feel very happy (positive mood group) or did not elicit any specific emotion (neutral mood group). The participants were then asked to close their eyes and to vividly imagine reliving the recalled experience. They were encouraged to use concrete visualizations and to 
take the requested field perspective while imagining (Watkins \& Moberly, 2009; based on Holmes et al., 2008). Participants then imagined the recalled experience for $30 \mathrm{~s}$ (twice), interrupted by questions about what they could see, hear, and feel (based on Watkins \& Moberly, 2009). Classical music fragments started to play during the memory recall and continued to play throughout the experimental sessions, such that the music would serve as an implicit trigger for the corresponding mood. In Experiment 1, the same (neutral) music samples were played in both mood groups so as to balance any possible interference effects created by the music on the recorded EEG signal. However, in order to elicit stronger moodinducing effects and a conditioned context that was better adjusted to the targeted mood, we used "happy" music in the positive mood condition and neutral music in the neutral mood condition in Experiment 2. ${ }^{1}$ These music samples had been validated in previous research (Bower \& Mayer, 1989; Mitterschiffthaler, Fu, Dalton, Andrew, \& Williams, 2007).

To measure subjective levels of mood, participants were asked to indicate how they felt using the Positive and Negative Affect Schedule (PANAS; Watson, Clark, \& Tellegen, 1988) and three 10 -cm horizontal visual analogue scales (VAS) for feelings of happiness, pleasantness, and sadness. The left anchor of each VAS was labeled Neutral, while the right one was labeled As happy/pleasant/sad as you can imagine.

Attention task We adapted a standard experimental paradigm (Rauss et al., 2009; Rossi \& Pourtois, 2012; Schwartz et al., 2005). The task was programmed using EPrime, Version 2 (Psychology Software Tools Inc., Sharpsburg, PA). The participants were instructed to carefully attend to a rapid serial visual presentation (RSVP) of short tilted lines $(1 \mathrm{~cm})$ at central fixation (Fig. 1). In Experiment 1, they silently counted the occurrences of deviant lines within each block and reported the total number at the end of the block, while in Experiment 2 they were instructed to press a key with their left finger whenever they could detect the occurrence of a deviant line. Deviant lines were tilted $45^{\circ}$, and standard lines $35^{\circ}$, counterclockwise from the vertical axis. The standard:target ratio was $4: 1$,

\footnotetext{
${ }^{1}$ We performed a control study $(n=15)$ to assess whether the music samples alone, without the MIP, could have influenced the behavioral results in Experiment 2. Therefore, participants performed a dual attention task while either positive music fragments (three blocks) or neutral music fragments (three other blocks) were played in the background. The participants were instructed to pay no specific attention to the music. No MIP was administered. The results showed that the neutral and positive music samples did not differentially influence accuracy or RTs for either the central task or the discrimination of peripheral textures, suggesting that the MIP, and not the use of different music samples alone, modulated attention allocation in the upper visual field (Exp. 2).
}

with the number of deviant lines varying between eight and 12 per block. Because previous studies had confirmed that this task requires central/foveal vision and sustained attention (see Rauss et al., 2009; Rossi \& Pourtois, 2012; Schwartz et al., 2005), we used it to ascertain that participants were fixating at the center of the screen, where these short lines were presented. Decoupled from this central RSVP stream, uniform visual textures were shown in the upper visual field at unpredictable locations (i.e., one of three locations) and times (i.e., a variable stimulus onset asynchrony [SOA] between central stimulus offset and peripheral texture onset) relative to the central stimuli (Fig. 1a and b). The experiment consisted of 300 central stimuli (i.e., 240 standard and 60 target lines) and 150 peripheral stimuli (i.e., 120 after the presentation of a central standard line, and 30 after a central target line). The peripheral stimuli consisted of two arrays of rectangular elements forming a homogeneous visual texture $\left(3^{\circ} \times 34^{\circ}\right.$ of visual angle $)$, briefly flashed in the upper visual field at $5.3^{\circ}$ (close), $7.8^{\circ}$ (medium), or $10.3^{\circ}$ (far) from central fixation (see Fig. 1a). Two different types of rectangular elements $(0.6 \mathrm{~cm}$ wide $\times$ $0.3 \mathrm{~cm}$ high [Type 1] or $0.5 \mathrm{~cm}$ wide $\times 0.4 \mathrm{~cm}$ high [Type 2]; see Fig. 1C) were used, with 25 presentations of each type at each location. These peripheral textures were task-irrelevant (i.e., distractors) in Experiment 1, as opposed to in Experiment 2, in which participants were invited to discriminate the actual content of visual textures (either Type 1 or 2, based on the actual rectangular elements; i.e., dual-task setting). In the latter experiment, a fixation cross was presented again at the offset of the visual texture and remained on the screen until participants pressed one of two keys using their right hand (the stimulus-response mapping was alternated across participants). A reminder of the stimulusresponse mapping was shown at the beginning of each block. The stimuli were gray and presented against a uniform black background. The participants were seated $57 \mathrm{~cm}$ in front of a 19-in. CRT screen, with their head movements restrained by a chinrest.

Localizer In order to identify the $\mathrm{C} 1$ and to obtain independent evidence that the earliest visual ERP component recorded during the main attention task for the peripheral textures genuinely corresponded to a retinotopic $\mathrm{C} 1$, we administered to the participants of Experiment 1 two extra blocks, of peripheral textures only, under passive viewing conditions at the end of the experimental session (see Rossi \& Pourtois, 2012). In these blocks, no RSVP stream was presented at fixation. In each block, 120 stimuli were presented in random order, with even numbers of presentations at six possible locations (upper or lower visual field and close, medium, or far from fixation). The localizer blocks were administered at the end of the experiment in order to avoid unwanted priming effects, since they remained fully 
a

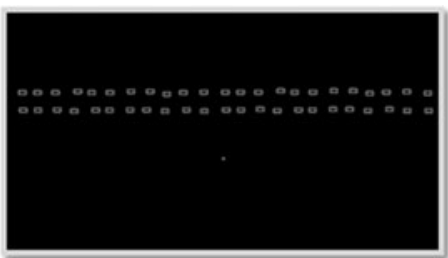

Close

b

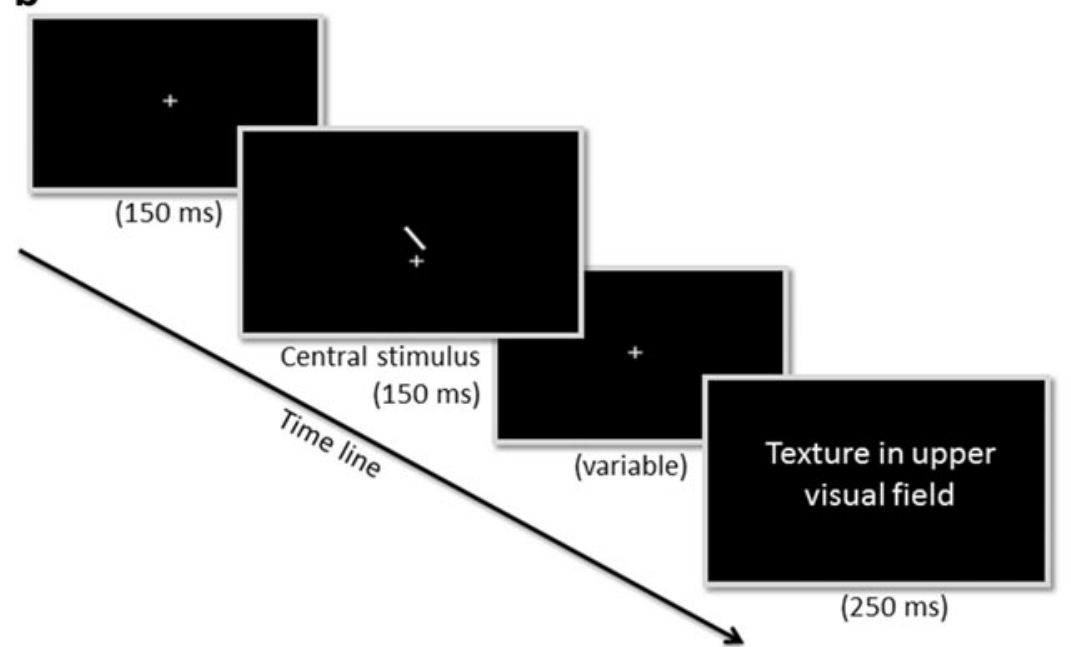

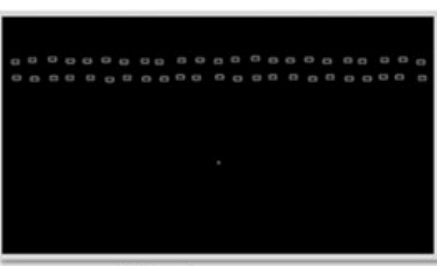

Medium

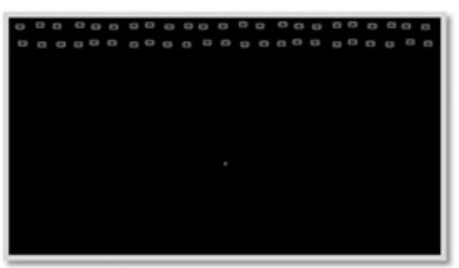

Far

C Type 1

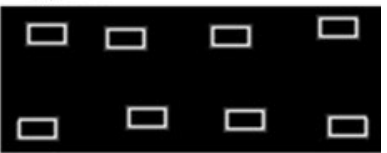

Type 2

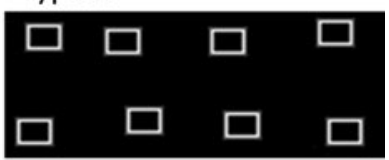

Fig. 1 Task. (a)Visual textures were briefly presented in a random order at one of three possible locations in the upper visual field: close to fixation, at a medium distance, or far from fixation. These textures had to be ignored during the EEG experiment, but were task-relevant in the behavioral experiment. In this case, participants were asked to judge whether the elements forming each texture were either more square or more asymmetric rectangles, while keeping fixation at the center of the screen. (b) The main task at fixation (shared across the two experiments) consisted of an oddball line orientation detection task. Participants were asked to detect, either covertly (EEG experiment) or overtly (behavioral experiment), deviant line orientations embedded in an RSVP stream at fixation. Standard lines were tilted $35^{\circ}$ counterclockwise, while deviant lines were tilted $45^{\circ}$ in the same direction. The ratio between the numbers of standard and target orientations presented was $4: 1$. The fixation cross after the line stimulus was shown

task-irrelevant in Experiment 1, in agreement with previous research (e.g., Rossi \& Pourtois, 2012). Moreover, residual effects of (positive) mood were expected to be minimal during these final blocks, because no repetition of the MIP took place prior to them.

\section{Procedure}

In Experiment 1, the participants were first prepared for EEG recording. They completed a practice session including two blocks of 20 trials (in total, 32 standard and eight target lines), which were repeated until $80 \%$ of the (deviant) central lines had been reported correctly. Next, a positive or neutral mood was induced by means of the MIP, which was briefly $(5 \mathrm{~min}$ ) repeated at the end of Block 3 in order to maintain the targeted mood throughout the experimental for $250-500 \mathrm{~ms}$, in the EEG experiment, and for 500-750 ms, in the behavioral experiment. On half of the trials (random order), a visual texture was briefly presented $(250 \mathrm{~ms})$ at one of three positions in the upper visual field, at a variable time following the presentation of the central line. On the other half, no texture was presented. Hence, the appearance and the actual location of the visual textures were unpredictable, and their presentations never overlapped with the centrally presented lines appearing on the screen roughly every $775 \mathrm{~ms}$ in the EEG experiment, and every 1,025 $\mathrm{ms}$ in the behavioral experiment. (c) Half of the peripheral textures were made up of one type of rectangular element $(0.6 \mathrm{~cm}$ wide $\times 0.3 \mathrm{~cm}$ high $)$, whereas the other half had slightly different rectangular elements $(0.5 \mathrm{~cm}$ wide $\times 0.4 \mathrm{~cm}$ high $)$, with equal numbers of presentations of each type for each of the three locations

session. Participants completed six different blocks of 50 trials each of the attention task. Each trial started with the presentation of a fixation cross $(250 \mathrm{~ms})$, followed by a central line $(150 \mathrm{~ms})$ at the same location (see Fig. 1b), and again a fixation cross, displayed for the duration of the SOA (the average duration in Exp. 1 was $375 \mathrm{~ms}$, randomly varying between 250 and $500 \mathrm{~ms}$; in Exp. 2, it was $625 \mathrm{~ms}$, randomly varying between 500 and $750 \mathrm{~ms}$ ). After the SOA, in half of the trials a visual texture was flashed for $250 \mathrm{~ms}$ at one of three possible locations in the upper visual field; in the other half of the trials, only the fixation cross remained on screen for the same interval. Trials were presented in a semirandom order. The first three trials in a block never contained a target line or a peripheral distractor. The instructions emphasized maintaining the highest accuracy possible for the task at fixation (Exps. 1- 
2), as well as both accuracy and speed for the twoalternative forced choice task performed with the peripheral textures (Exp. 2). In addition, the instructions given to the participants of Experiment 2 clearly emphasized the importance of a high accuracy for the primary task at fixation and the need to maintain attention focused at this central location throughout the whole experiment. VASs and the PANAS were administered at the beginning of the experiment (baseline measure), after each MIP, and at the end of the experiment in order to observe possible changes in mood before, during, and after the MIP.

Once the experimental session was completed, participants filled out four trait-related/personality questionnaires: the Beck Depression Inventory (BDI; Beck, Steer, Ball, \& Ranieri, 1996), the Spielberger State-Trait Inventory, Trait version (STAI-T; Spielberger, Gorsuch, Lushene, Vagg, \& Jacobs, 1983), the BIS/BAS scales (Carver \& White, 1994), and the Spontaneous Use of Imagery Scale (SUIS; Reisberg, Pearson, \& Kosslyn, 2003).

To sum up, in both Experiments 1 and 2 participants performed a main oddball detection task at fixation, whereas peripheral textures were briefly flashed during the interstimulus interval in the upper visual field at unpredictable times and locations. However, in Experiment 2, these peripheral textures were task-relevant and required an overt visual discrimination, while in Experiment 1 (EEG) they remained task-irrelevant. Additional methodological differences between the two experiments concerned (1) the music played in the background during the experiment (i.e., the same, neutral samples for both groups in Exp. 1 vs. neutral or positive samples in Exp. 2), and (2) the specific task demands for the central stimuli (i.e., in Exp. 1, the deviant lines had to be detected and counted silently by the participants before reporting the total number at the end of each block, whereas in Exp. 2, participants had to make a specific keypress online whenever they detected a deviant line).

\section{Analyses of behavioral data}

Changes in mood were verified by comparing post- to preMIP subjective measures of affect. We first computed postMIP average scores for each VAS and the PANAS administered after the two MIPs and at the end of the experiment. Next, we performed a 2 (time: baseline vs. post-MIP) $\times 2$ (mood: neutral vs. positive) mixed ANOVA on the VAS ratings and the PANAS scores, followed by independent $t$ tests (planned comparisons). For the four trait-related questionnaires, the mean scores were calculated and compared between groups in order to check for possible uncontrolled personality differences between the groups.

In Experiment 1, accuracy for the oddball task at fixation was computed for each participant separately by subtracting the reported number from the actual number of deviant lines, and the sum of these deviations was computed for each of the six blocks. Next, these numbers were compared between groups using an independent Student's $t$ test. In Experiment 2, accuracy and reaction times (RTs) for the central task were compared between groups using independent $t$ tests. Performance for the peripheral textures (accuracy and RTs) was analyzed using mixed ANOVAs, with the within-subjects factor Distance (close, medium, or far) and the between-subjects factor Mood (positive vs. neutral). Trials with errors on the central task, as well as those with RTs exceeding $\pm 2.5 S D$ s above or below the individual mean RT on both the central and peripheral tasks, were excluded from further analysis. The exclusion rates did not differ between the two mood groups (positive, $M=9.57 \%, S D=3.70$; neutral, $M=7.94 \%, S D=3.48), t(33)=1.34, p=.19$. Statistical analyses were run on $91.22 \%$ of the total data.

Greenhouse-Geisser correction was applied when the assumption of sphericity was violated. In these cases, we report corrected $p$ values and uncorrected degrees of freedom.

\section{EEG data acquisition and reduction}

EEG data were recorded from 128 electrodes placed according to the extended 10-20 EEG system using an elastic cap (Biosemi Active Two System). Vertical and horizontal eye movements were recorded by means of additional bipolar electrodes placed, respectively, above and below the left eye and on the outer canthi of both eyes. EEG signals were referenced online to the CMS-DRL ground and continuously sampled at $512 \mathrm{~Hz}$.

EEG signals were referenced offline to the linked mastoids, using Brain Vision Analyzer 2.0 (Brain Products $\mathrm{GmbH}$, Munich, Germany). Band-pass filters between 0.016 and $70 \mathrm{~Hz}$ and a notch filter $(50 \mathrm{~Hz})$ were used. The filtered data were then segmented into stimulus-locked epochs using a segmentation window from $100 \mathrm{~ms}$ pre- to 800 ms post-stimulus-onset. Eye-blink artifacts were automatically corrected by means of the standard procedure put forward by Gratton and colleagues (Gratton, Coles, \& Donchin, 1983), and individual epochs were baseline corrected using the entire prestimulus interval. Epochs of the EEG containing residual artifacts exceeding $\pm 75 \mu \mathrm{V}$ were semiautomatically rejected. Noisy electrodes were interpolated using a spherical spline procedure, and we computed individual averages separately for each condition. Finally, a $30-\mathrm{Hz}$ low-pass filter was applied before the calculation of grand average waveforms.

To characterize and analyze ERPs to the peripheral distractors, we performed a detailed ERP topographic mapping analysis. The added value and underlying principles of this data-driven analysis have been described extensively elsewhere (Michel, Seeck, \& Landis, 1999; Murray, Brunet, \& 
Michel, 2008; Pourtois, Dan, Grandjean, Sander, \& Vuilleumier, 2005; Pourtois et al., 2008; Pourtois, De Pretto, Hauert, \& Vuilleumier, 2006; Pourtois, Thut, Grave de Peralta, Michel, \& Vuilleumier, 2005). Since the C1 is primarily a location-sensitive early visual ERP, the exact same stimulus would elicit a different topography and strength of the $\mathrm{C} 1$ electric field depending on its actual position in the peripheral visual field (Clark et al., 1995). Accordingly, we used a standard ERP topographic mapping analysis that was able to capture these changes in the expression of the electric field (i.e., topography) of the $\mathrm{C} 1$ as a function of stimulus position. Using a $K$-means spatial cluster analysis, we first identified in the grand average data the dominant scalp topographies corresponding to the $\mathrm{C} 1$ (95$115 \mathrm{~ms}$ post-stimulus-onset) and $\mathrm{P} 1$ (165-185 ms poststimulus-onset) components generated in response to the peripheral distractors, as well as the P300 (400-600 ms post-stimulus-onset) in response to the centrally presented line stimuli. The following standard parameters were used: average reference, 100 random trials, smoothing strength (Besag factor) of 10, smoothing half-window size of 3, merging of clusters correlated above .92 , rejection of segments less than or equal to three time frames, and no sequentializing. These topographic maps were determined objectively using both cross-validation (Pascual-Marqui, Michel, \& Lehmann, 1995) and the Krzanowski-Lai criteria (Pascual-Marqui et al., 1995; Tibshirani, Walther, \& Hastie, 2001). Following standard practice, these dominant scalp topographies were then fitted back to the ERPs of each individual participant using spatial-fitting procedures to quantitatively determine their representations across participants and conditions. The global explained variance (GEV, or goodness of fit) was then used as the main dependent variable in standard parametric statistical analyses of variance (ANOVAs) to verify, on the one hand, whether mood and position reliably influenced the $\mathrm{C} 1$ and/or the $\mathrm{P} 1$ in response to the peripheral textures, and on the other hand, whether mood and stimulus type (standard or target) modulated the P300 in response to the task-relevant stimuli.

\section{Results}

\section{Experiment 1}

\section{Changes in mood: Manipulation check}

The 2 (time) $\times 2$ (mood) mixed ANOVA on the VAS scores showed a significant interaction effect between time and mood for feelings of happiness [baseline: positive, $M=$ 4.48, $S D=2.83$; neutral, $M=4.80, S D=2.92$; post-MIP: positive, $M=7.57, S D=1.16$; neutral, $M=4.32, S D=3.00$; $F(1,31)=23.83, p<.001]$ and pleasantness [baseline: positive, $M=4.89, S D=2.72$; neutral, $M=4.30, S D=3.13$; post-MIP: positive, $M=7.57, S D=1.12$; neutral, $M=4.66$, $S D=3.00 ; F(1,31)=10.21, p=.003]$. Next, we compared the VAS scores between the positive and neutral mood groups, separately at baseline versus post-MIP. Independent $t$ tests showed, as expected, no difference for reported happiness $[t(31)=0.32, p=.75]$ or pleasantness $[t(31)=0.43, p=.56]$ between the mood groups at baseline. However, post-MIP mood measurements showed significant differences between the positive and neutral mood groups for feelings of happiness $[t(31)=4.14, p<.001]$ and pleasantness $[t(31)=3.78, p=.001$; see Fig. 2]. The 2 (time) $\times 2$ (mood) mixed ANOVA for the sadness VAS did not show a significant interaction effect [baseline: positive, $M=0.73, S D=0.97$; neutral, $M=0.44, S D=0.54$; post-MIP: positive, $M=0.51, S D=0.73$; neutral, $M=0.34$, $S D=0.40 ; F(1,31)=0.44, p=.51]$. These results confirmed a significant and selective increase in positive affect after the MIP in the positive mood group, but no such change in the neutral mood group.

The 2 (time) $\times 2$ (mood) mixed ANOVA on the PANAS scores showed a significant interaction effect between the time and mood groups for the Positive Affect (PA) scales [baseline: positive, $M=32.65, S D=5.53$; neutral, $M=32.88$, $S D=5.26$; post-MIP: positive, $M=34.96, S D=4.63$; neutral, $M=30.54, S D=7.28 ; F(1,31)=8.69, p=.006]$, but not for the Negative Affect (NA) scales [baseline, $M=23.56$, $S D=5.81$; post-MIP, $M=10.89, S D=1.86 ; F(1,31)=1.24$, $p=.27]$. An independent $t$ test (PA scales) showed a significant difference between the positive and neutral mood groups post-MIP $[t(31)=2.09, p=.04]$. At baseline, this difference was not significant $[t(31)=0.54, p=.90]$.

\section{Behavioral results}

On average, the participants in both mood groups had low error rates (sum of absolute deviations in positive group, $M=4.29, S D=2.78$; neutral group, $M=4.88, S D=5.60$ ). No significant group difference in accuracy emerged $[t(31)=0.38, p=.71$; see Fig. $3 b]$, suggesting that behavioral performance for the central task was high and balanced between the two mood groups.

\section{ERP results}

P300 The topographical mapping analysis identified three main clusters/topographies during the P300 time interval following the onset of the central tilted lines. We compared the mean GEV values obtained for these three dominant maps using a mixed ANOVA, with Stimulus Type (standard vs. target) and Map Configurations $(n=3)$ as within-subjects factors and Mood (positive vs. neutral) as a between-subjects factor. The results showed a significant effect of stimulus type 

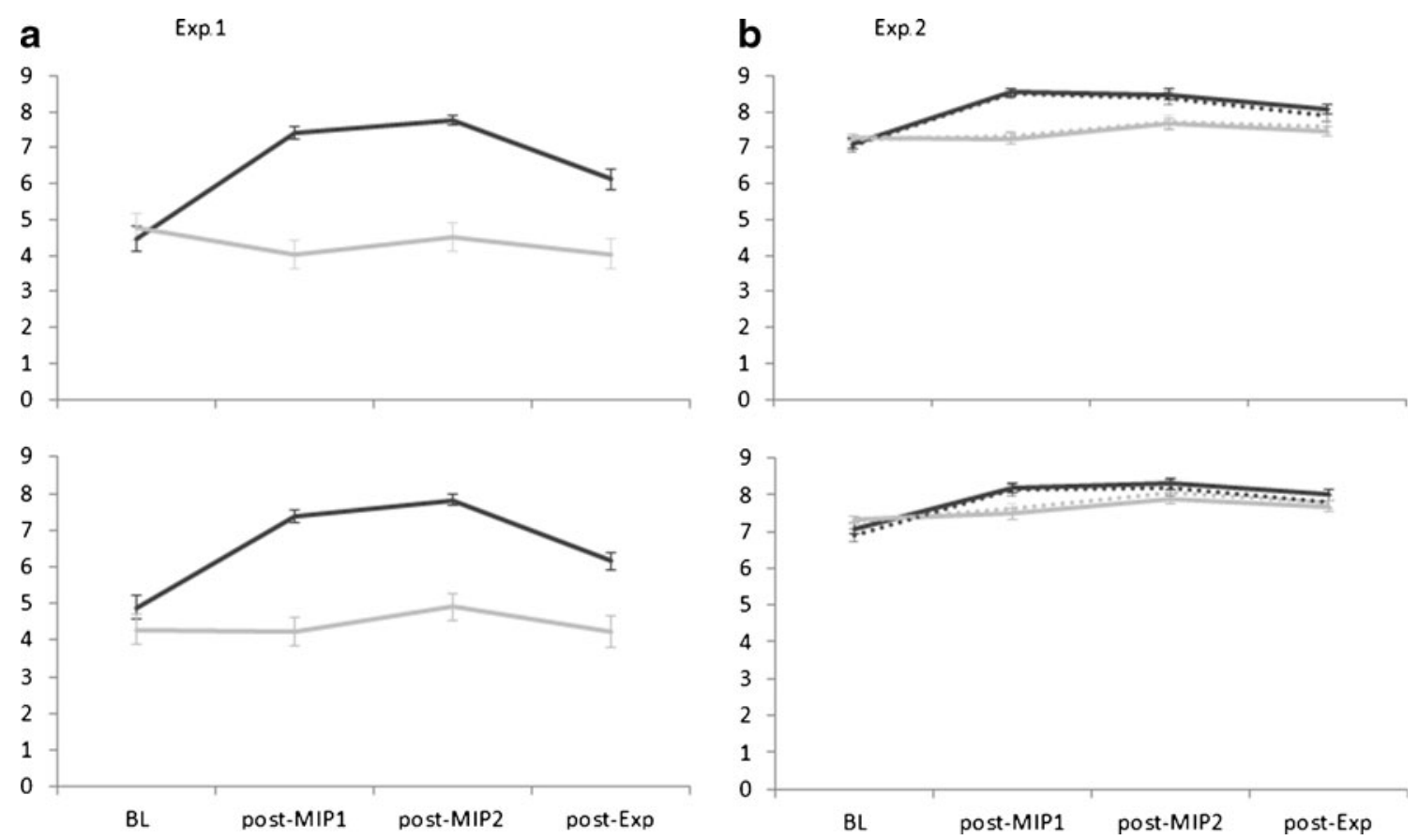

Fig. 2 Evolution of levels of positive mood across the four (consecutive) measurement points, presented separately for the positive mood group (dark gray lines) and the neutral mood group (light gray lines). Mean scores (with error bars representing 1 SEM) for the happy VAS (upper panels) and the pleasant VAS (lower panels) are presented

$[F(1,31)=4.385, p<.05]$ and a significant interaction effect between stimulus type and map configuration $[F(2,62)=$ $15.180, p<.001]$, showing that the configuration of the P300 substantially changed depending on the stimulus type (see Fig. 4), in line with previous research (Kim, Kim, Yoon, \& Jung, 2008; Kok, 2001; McCarthy \& Donchin, 1981;

for (a)Experiment 1 and (b)Experiment2. For Experiment2, the results are shown for all 35 participants (continuous lines) versus the 28 participants (14 per group) showing clear effects of mood depending on the mood induction procedure (dotted lines; see note 2)

Sawaki \& Katayama, 2007). Importantly, no significant main effect of mood was found $[F(1,31)=0.016, p=.90]$, nor a significant interaction effect between mood and map configuration $[F(2,62)=0.035, p=.96]$, indicating that participants in both groups processed standard versus target central stimuli differentially.

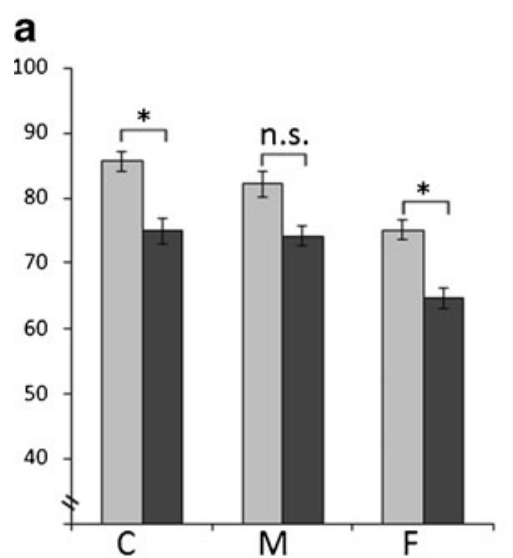

Fig. 3 (a) Accuracy rates for the identification of peripheral textures (Exp.2) show a decrease in accuracy with increasing distance from fixation ( $\mathrm{C}$, close to fixation; $\mathrm{M}$, medium position; $\mathrm{F}$, far from fixation). However, overall lower accuracy was found in the positive mood group (dark gray) than in the neutral mood group (light gray). (b) The left panel shows accuracy rates for the oddball line orientation task at fixation for the EEG experiment. Trials on which participants correctly
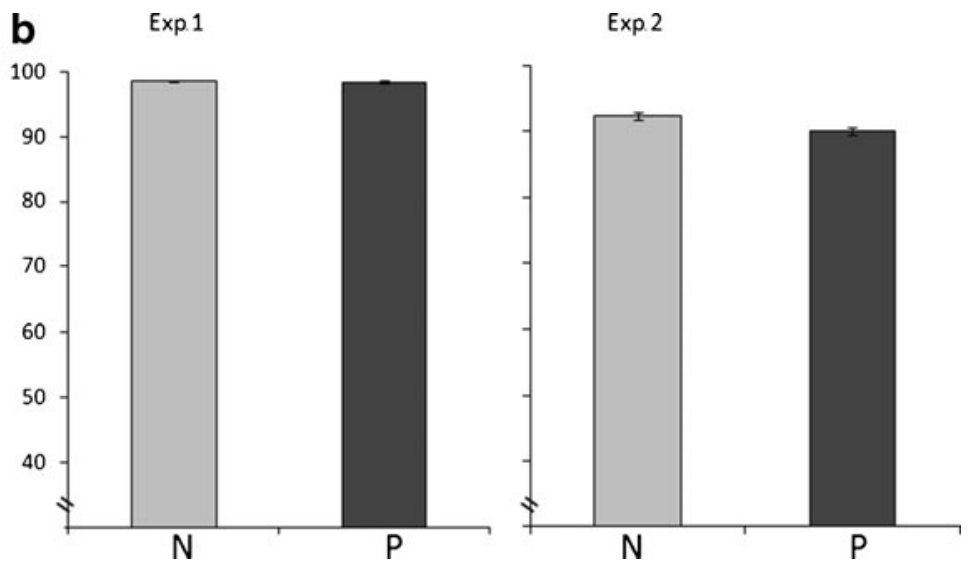

detected a target (or correctly rejected a nontarget) were counted, and these numbers were then converted to percentages (bearing in mind that, in total, 300 trials were presented). The right panel shows the results for the behavioral experiment. In each experiment, no accuracy difference was found between the neutral $(\mathrm{N})$ and positive $(\mathrm{P})$ mood groups. ${ }^{*} p<.05$; black bars represent standard errors of the means 


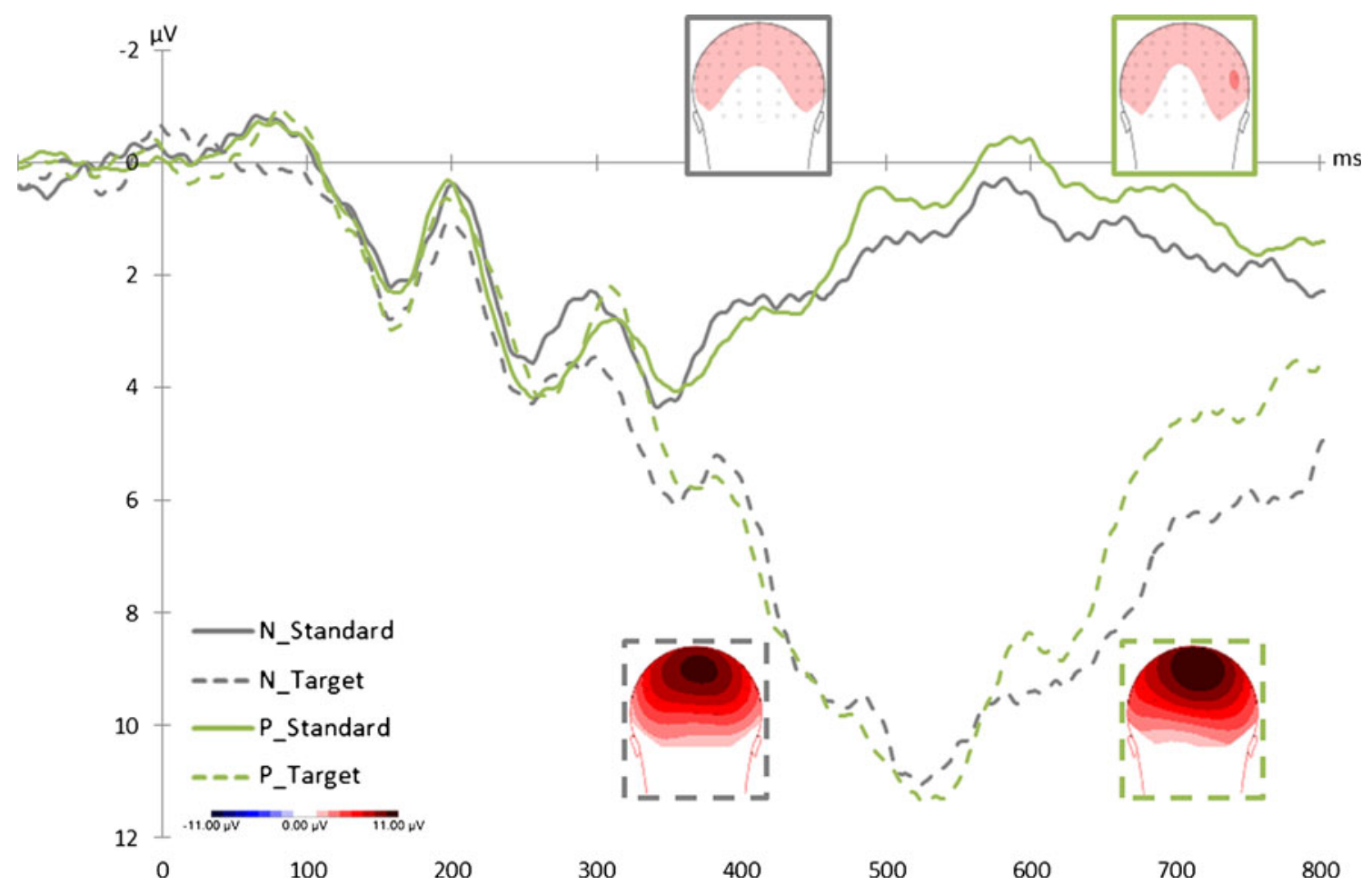

Fig. 4 Grand average ERPs to standard (solid lines) versus target (dashed lines) central stimuli at a representative midline electrode $(\mathrm{POz})$. A clear P300 is present for target line orientations in the neutral (gray) and positive (green) mood groups, whereas this decision-related component was strongly attenuated for standard line orientations. This

C1 Two main topographies were found during the $\mathrm{C} 1$ time window (95-115 ms; see Fig. 5a) following the onset of the peripheral textures. A mixed ANOVA with Map Configurations $(n=2)$ and Distance (close, medium or far) as within-subjects factors and Mood (positive vs. neutral) as a between-subjects factor was performed on the GEV values extracted for these two $\mathrm{C} 1$ topographies. The results showed a significant main effect of distance $[F(2,62)=7.737, p=.001]$ and a significant interaction effect between distance and $\operatorname{mood}[F(2,62)=$ $8.035, p=.001]$. Whereas the former effect confirmed that the topography of the $\mathrm{C} 1$ component reliably changed depending on the position of the stimulus in the upper visual field, the latter effect suggested that the induced positive mood influenced this position-specific encoding of the distractor (as reflected by the $\mathrm{C} 1$ topography). Planned comparisons revealed a significant drop of the GEV of the dominant $\mathrm{C} 1$ map (i.e., being maximum for the position closest to fixation) as a function of distance in the neutral mood group [close vs. medium, $t(15)=2.71, p=.02$; close vs. far, $t(15)=$ $2.50, p=.03]$, consistent with a nonlinear spatialgradient effect. However, this effect was not observed for the $\mathrm{C} 1 \mathrm{~s}$ of participants in the positive mood group [close vs. medium, $t(16)=0.39, p=.70$; close vs. far, conspicuous P300 effect reflected attention allocation to the target stimuli and was similar for the neutral and positive mood groups (see the text for the numerical values). Topographical voltage maps (back views) for the $\mathrm{P} 300$ component (computed 400-600 ms post-stimulusonset) are shown for the two conditions and two groups, separately

$t(16)=1.20, p=.25$; see Fig. $5 \mathrm{~b}$ and $\mathrm{c}]$. In this group, the explained variances of the dominant $\mathrm{C} 1$ topography were not significantly different across the three spatial positions, suggesting a broadening of attention. Together, these results suggest a link between changes in positive mood and position-dependent early brain responses in V1 to these (unattended) textures shown in the upper visual field.

Three dominant maps were found during the $\mathrm{C} 1$ time window (95-115 ms) for the ERP data recorded during the localizer (upper visual field, see Fig. 6), whereas two dominant maps were found for the $\mathrm{C} 1 \mathrm{~s}$ recorded during the main task. Presumably, this discrepancy may tentatively be explained by the different task demands (and stimulus parameters for the central stimuli) between these two sessions. Because the peripheral textures used in the localizer and in the main task were identical but embedded in different experimental contexts, it is likely that the topographical segmentation data analysis identified slight differences in the actual expressions of the $\mathrm{C} 1$ map across these two sessions.

A mixed ANOVA with Map Configurations $(n=3)$ and Distance (close, medium, or far) as within-subjects factors and the between-subjects factor Mood (positive vs. neutral) was performed on the GEV values extracted for these maps 
(localizer). The ANOVA showed a significant interaction effect between distance and map $[F(2,62)=7.71, p<$ $.001]$, while the mood by map $[F(2,62)=0.758, p=.47]$ and mood by distance $[F(2,62)=1.53, p=.22]$ interactions were nonsignificant, suggesting similar early retinotopic encodings of the peripheral textures in both groups. These results suggest that, unlike the $\mathrm{C} 1$ recorded during the main attention task, the $\mathrm{C} 1$ elicited by the same peripheral distractors during the localizer run (passive viewing) was not reliably influenced by mood. Importantly, a systematic comparison of topographies and waveforms for the $\mathrm{C} 1 \mathrm{deflec}-$ tion recorded during the localizer versus the main attention task for the same peripheral textures confirmed that this early negative component unambiguously corresponded to a genuine $\mathrm{C} 1$ deflection, showing the expected polarity reversal as a function of lower versus upper visual presentation (see Fig. 6).

P1 During the time interval of the P1 (165-185 ms) following the onset of the peripheral textures (main task), a single dominant topographical component was found to account for the ERP signal across the three positions (close, middle, or far; see Fig. 5a). A mixed ANOVA with Distance (close, medium, or far) as a within-subjects factor and Mood (positive vs. neutral) as a between-subjects factor on the GEV values extracted for this dominant P1 topography did not show any systematic variation with position of the peripheral textures $[F(2,62)=2.06, p=.14]$, confirming that this extrastriate component was most likely responding to the content, rather than the position, of these stimuli. Moreover, this analysis showed no significant main effect of mood $[F(1,31)=1.11, p=.30]$, nor a significant interaction effect between mood and distance $[F(2,62)=0.85, p=.43]$.

\section{Questionnaires}

A set of $t$ tests performed on the questionnaire data failed to reveal any significant group differences [BDI, $t(31)=0.29$, $p=.78$; STAI-T, $t(31)=0.30, p=.77$; SUIS, $t(31)=0.08$, $p=.23$; BIS/BAS scales: BIS, $t(31)=0.53, p=.60 ; \mathrm{BAS}$ Drive, $t(31)=0.61, p=.55$; BAS Fun, $t(31)=0.12, p=.91$; BAS Reward, $t(31)=0.09, p=.93]$, suggesting that the observed ERP differences for the processing of the peripheral textures between the two groups could not be explained by uncontrolled group differences in these specific personality traits.

\section{Experiment 2}

\section{Changes in mood: Manipulation check}

The 2 (time) $\times 2($ mood $)$ mixed ANOVA on the VAS scores showed a significant interaction effect between these two
Fig. 5 (a)Results of the topographical ERP mapping analysis (EEG experiment). This analysis identified a main cluster (88-127 ms poststimulus-onset for the close position) corresponding to the striate $\mathrm{C} 1$ component, followed by another one corresponding to the extrastriate P1 component (141-186 ms post-stimulus-onset for the close position). Whereas the topography of the $\mathrm{C} 1$ substantially changed with the position (C, close; $\mathrm{M}$, medium; $\mathrm{F}$, far) of the (unattended) textures in the upper visual field, the configuration of the electric field of the P1 did not (see the text for the numerical values). The corresponding voltage maps (back views) are also shown. Following standard practice, amplitude differences were normalized (i.e., the amplitude value at each electrode was divided by the global field power). (b)Grand average ERPs at electrode POz to visual textures, separately for each group (either neutral mood [left panel] or positive mood [right panel]) and each position (close [solid lines], medium [dashed lines], or far [dotted lines]) in the upper visual field. As expected, the amplitude of the $\mathrm{C} 1$ varied substantially as a function of the position of the textures in the upper visual field (i.e., smaller for far relative to close position), but this effect was stronger for participants in a neutral mood as compared to those in a positive mood. (c)Results of the back-fitting (see the Method section) of the dominant $\mathrm{C} 1$ topography (see the $\mathrm{C} 1$ frame in panel a). Whereas the goodness of fit of the dominant $\mathrm{C} 1$ topography sharply decreased for the medium and far, relative to the close, positions in the neutral mood group (left panel) - suggesting a normal spatial-gradient effect - this effect was not seen in the positive mood group (right panel), in whom $\mathrm{C} 1$ activity was not significantly lower for the far and medium positions than for the close position (see the text for the numerical values). ${ }^{*} p<.05$; black bars represent standard errors of the means

factors for feelings of happiness [baseline: positive, $M=$ 7.11, $S D=1.29$; neutral, $M=7.28, S D=0.87$; post-MIP: positive, $M=8.37, S D=1.04$; neutral, $M=7.46, S D=1.12$; $F(1,33)=15.07, p<.001]$ and pleasantness [baseline: positive, $M=7.08, S D=1.25$; neutral, $M=7.32, S D=$ 0.83; post-MIP: positive, $M=8.16, S D=1.06$; neutral, $M=$ 7.69, $S D=1.10 ; F(1,33)=4.51, p=.04$; see Fig. 2], but not for feelings of sadness [baseline: positive, $M=1.33, S D=$ 1.56; neutral, $M=0.75, S D=0.66$; post-MIP: positive, $M=$ $0.61, S D=0.55$; neutral, $M=0.58, S D=0.41 ; F(1,33)=$ $2.41, p=.13$ ]. Planned comparisons confirmed that there was no significant group difference at baseline in reported feelings of happiness $[t(33)=0.450, p=.51]$, pleasantness $[t(33)=0.665, p=.656]$, or sadness $[t(33)=$ $1.409, p=.17]$. Post-MIP VAS scores differed significantly between the positive and neutral mood groups for feelings of happiness $[t(33)=2.481, p=.018]$, but the difference did not reach significance for pleasantness $[t(33)=1.306, p=.20]$ or sadness $[t(33)=0.215, p=.83]$. Altogether, these results confirmed an increase in happiness after the MIP in the positive mood group, but no change in the neutral mood group.

By contrast, the 2 (time) $\times 2$ (mood) mixed ANOVA on the PANAS scores showed no significant interaction effect for the PA scales [baseline, $M=32.40, S D=5.41$; post-MIP, $M=32.19, S D=5.44 ; F(1,33)=1.97, p=.17]$ or for the NA scales [baseline, $M=12.34, S D=2.33$; post-MIP, $M=$ $11.26, S D=1.56 ; F(1,33)=0.28, p=.60]$. 


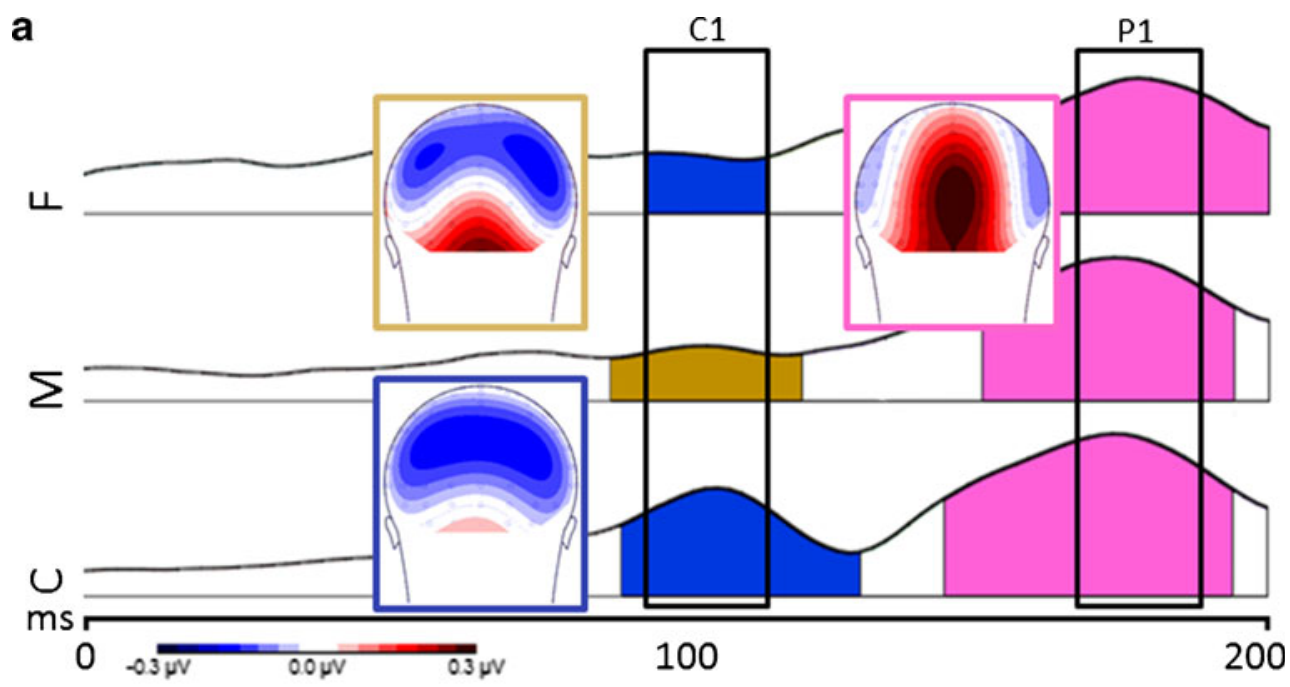

b
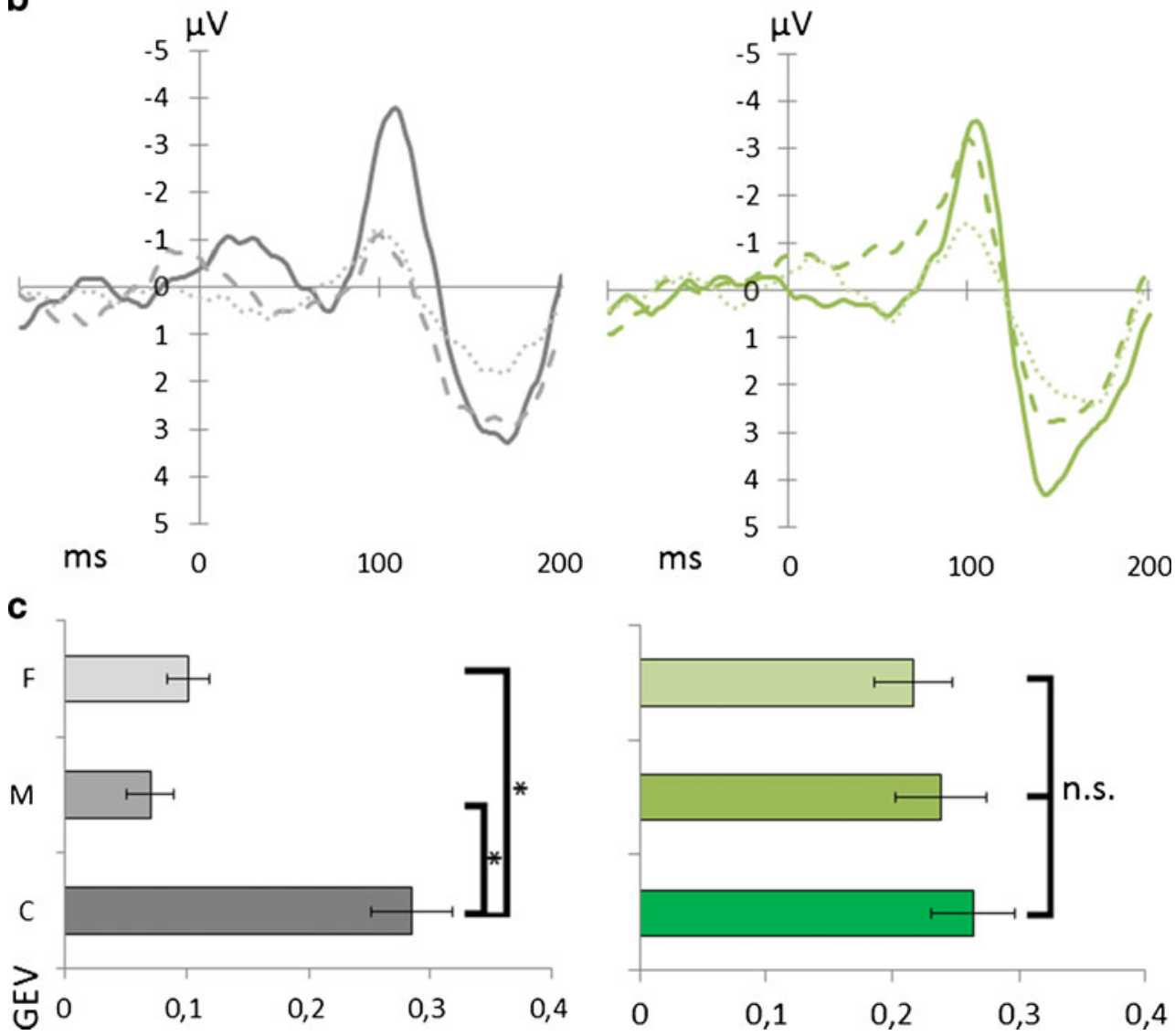

\section{Attention task}

Central stimuli The participants' accuracy was high in both groups (positive, $88.65 \%, S D=6.66$; neutral, $91.84 \%, S D=$ $6.05)$. We found no significant difference between the two groups in accuracy $[t(33)=1.49, p=.15$; see Fig. $3 b]$. The amounts of neither false alarms [positive, $M=4.89, S D=$
9.06; neutral, $M=2.41, S D=3.62 ; t(33)=1.05, p=.30]$ nor omissions [positive, $M=22.17, S D=10.83$; neutral, $M=$ $17.71, S D=10.39 ; t(33)=1.24, p=.22]$ differed significantly between the two groups. Likewise, RTs for correct detections were balanced between groups [positive, $M=$ 499.70, $S D=97.68$; neutral, $M=456.22, S D=61.66$; $t(33)=1.56, p=.13]$. 
Fig. 6 Results of the localizer run: Grand average ERPs at electrode $\mathrm{POz}$ to peripheral visual textures presented close to fixation, shown separately for the upper (blue line) and lower (red line) visual fields for (a) the neutral mood group and (b) the positive mood group. These ERPs confirm that in both groups a diagnostic $\mathrm{C} 1$ polarity reversal was equally present early following stimulus onset over occipito-parietal electrodes along the midline for visual stimulations in the upper versus the lower visual field, consistent with the electrophysiological signature of this early retinotopic visual component (Rauss etal., 2009). The corresponding voltage maps (back views) are shown
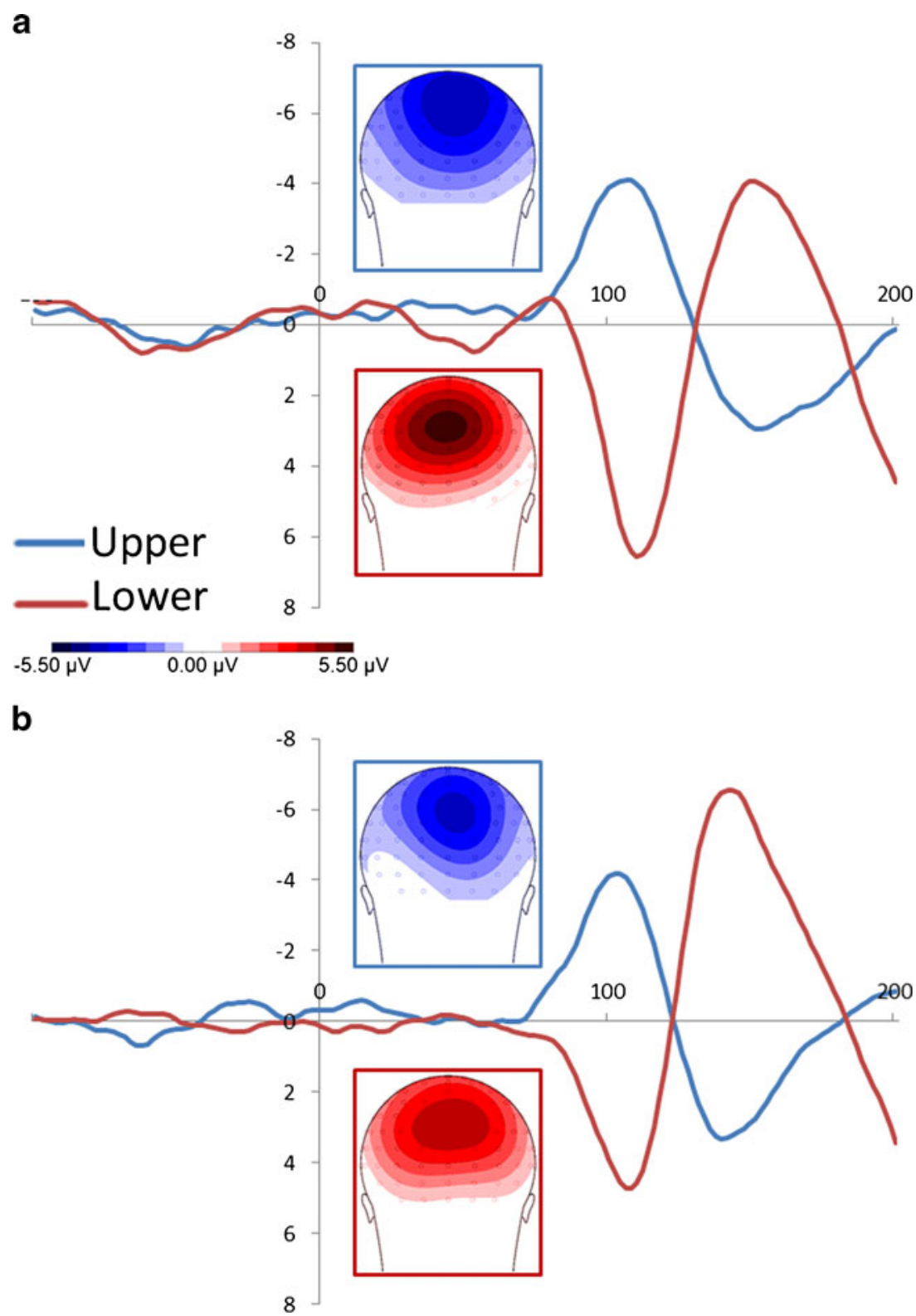

These results confirmed that participants correctly attended and maintained fixation to the central RSVP, and that positive mood induction did not simply affect the processing of these centrally presented lines, relative to neutral mood.

Peripheral stimuli The 2 (mood: neutral vs. positive) $\times 3$ (distance: close, medium, or far) mixed ANOVA performed on the mean accuracy scores revealed a significant main effect of distance $[F(2,66)=16.10, p<.001]$, showing, as expected, a monotonic decrease of performance as a function of distance relative to fixation (Fig. 3a): close position, $M=80.14, S D=15.62$; medium position, $M=78.03, S D=$ 15.14; far position, $M=69.96, S D=14.33$. Accuracy differed significantly between the far and medium positions $[t(33)=4.13, p<.001]$, as well as between far and close $[t(33)=4.83, p<.001]$. However, mean accuracy did not differ significantly between close and medium $[t(33)=1.41$, $p=.17]$. Importantly, this analysis also revealed a significant main effect of mood, indicated by overall lower accuracy in the positive mood group $(M=71.22, S D=12.57)$ than in the neutral mood group $(M=80.15, S D=13.11)[F(1,33)=2.23$, $p=.03$; see Fig. 3a]. The interaction effect between mood and distance was not significant $[F(2,66)=0.36, p=.70]$, indicating that the effects of mood did not differ across the three positions. Planned comparisons showed a significant difference between the positive and neutral mood groups for far $[t(33)=2.43, p=.02]$ and close $[t(33)=2.13, p=.04]$ positions, but no such difference between positive and neutral mood groups for the medium position $[t(33)=1.61$, 
$p=.12]$. Hence, accuracy with the peripheral textures was overall lower for participants in a positive mood, relative to a neutral mood. This result may be interpreted as a general drop in attentional selectivity for the textures shown in the upper visual field among participants with a positive mood. $^{2}$

Analysis of the RTs for correct responses confirmed that distance reliably influenced speed in a predictive way $[F(2,66)=13.49, p<.001]$, with faster decisions for peripheral textures shown close to fixation $(M=517.73$, $S D=136.01)$, relative to the medium position $(M=564.06$, $S D=155.62)[t(34)=3.72, p<.001]$ or the far position $(M=582.87, S D=151.21)[t(34)=4.49, p<.001]$. The RT difference between medium and far was not significant $[t(33)=1.44, p=.16]$. We did see a significant interaction effect between mood and distance $[F(2,66)=3.13, p=.05]$, showing a monotonic increase of RTs with increasing distance from fixation in the neutral mood group, but not for the positive mood group. No main effect of mood was found $[F(1,36)=1.04, p=.32]$.

\section{Questionnaires}

Comparisons performed on the personality questionnaire data failed to reveal any significant difference between the two mood groups [BDI, $t(33)=0.25, p=.80$; STAI-T, $t(33)=0.39, p=.70$; SUIS, $t(33)=0.25, p=.80$; BIS/BAS scales: BIS, $t(33)=0.26$, $p=.80$; BAS Drive, $t(33)=0.31, p=.76$; BAS Reward Responsiveness, $t(33)=0.82, p=.42$ ], except for the BAS Fun Seeking scale, with a (marginally) significantly higher score in the neutral $(M=6.59, p=1.12)$ than in the positive $(M=5.44$, $p=2.09)$ mood group $[t(33)=2.00, p=.054]$.

\section{Discussion}

The results of our study showed that positive mood can alter the earliest cortical stage of stimulus processing, presumably taking place in V1 (i.e., the $\mathrm{C} 1$ component; Rauss et al., 2011). More specifically, our ERP findings showed that the expression (topography) of the $\mathrm{C} 1$ to unattended peripheral distractors shown in the upper visual field strongly varied according

\footnotetext{
${ }^{2}$ When we analyzed the changes in levels of positive mood following the MIP (taking into account VAS and PANAS scores concurrently), we found that seven out of 35 participants showed an unusual pattern. Three of the participants assigned to the neutral mood group showed an increase in positive mood, while four participants assigned to the positive mood group showed a weak or no increase in positive mood following the MIP. We performed additional, refined statistical analyses excluding the data of these seven participants, but we did not observe any change in performance for the central task [the effect of group was still nonsignificant; $t(26)=1.18, p=0.25$ ], nor in visual discrimination of the peripheral textures [the effect of group was still significant; $F(1,26)=4.50, p<.05]$.
}

to the position (close, medium, or far) of these textures relative to central fixation. However, this early spatial-gradient effect was clearly altered during positive, relative to a neutral, mood. In line with a broadening of spatial attention with positive emotion, the dominant topography of the $\mathrm{C} 1$ was equally strong, regardless of the position of the texture in the upper visual field, for participants experiencing positive affect, whereas the $\mathrm{C} 1 \mathrm{~s}$ of participants in the neutral mood condition showed a clear and sharp topographical change according to the same manipulation (see Fig. 5b and c). Importantly, these results were obtained even though mood did not influence performance and decision-related ERP responses (i.e., P300) to the centrally presented visual stimuli, suggesting that an enhanced level of positive affect primarily had an influence on covert attention allocation toward peripheral (unattended), as opposed to central (attended), stimuli. We discuss the implications of these new findings below.

\section{Positive emotion broadens attention}

In order to assess the influence of positive mood on attentional processes, participants were randomly assigned to either a positive or a neutral MIP. Given that this MIP consisted of self-relevant imagery and music, the observed changes in positive affect may be attributed to the modulation of higher-level cognitive or affective processes, as opposed to mere phasic reward (Hickey, Chelazzi, \& Theeuwes, 2010; Kringelbach \& Rolls, 2004; Pessoa \& Engelmann, 2010; Rolls, 2000; van Steenbergen et al., 2009) or the selective induction of approach-motivated affect (Gable \& Harmon-Jones, 2008, 2010). In contrast, the novel MIP that we used elicited a positive mood characterized by low intensity in approach motivation, since the emotions evoked were not relevant in terms of any specific (task) goal. ${ }^{3}$ The results of the manipulation check showed that the MIP was successful in increasing subjective feelings

\footnotetext{
${ }^{3}$ Our results suggest that nonverbal VASs may be more sensitive than the PANAS for capturing subtle changes in positive mood (Rossi \& Pourtois, 2011), given that in Experiment 2, the pre-post change in mood was only found with the VASs. As the change in affective state after our MIP was mild, such a change might have been better captured by an instrument that can pick up small variations along a continuous scale (cf. VAS), as opposed to a limited number of discrete categories (cf. PANAS). Moreover, the presence of verbal labels for the PANAS may have prevented participants from deviating substantially in their estimations in a repeated measures design. Moreover, the Positive Affect scale of the PANAS principally measures "positively valenced affects" (Watson, Wiese, Vaidya, \& Tellegen, 1999) high in activation (Russell \& Carroll, 1999), as well as how much the participant enjoys engaging with his/her environment (Crawford \& Henry, 2004). By contrast, the VASs that we used in this study assessed current positive feelings, regardless of their origin/nature and independently from the levels of activation that they might elicit. Given the individualization of our MIP, the general scope of the VASs might have been better suited than the PANAS to capturing subtle changes in mood.
} 
of positive mood selectively in the positive mood group. However, our manipulation check did not include complementary measures of arousal. Hence, it is possible that not only positive valence, but also arousal, accounted for the changes in attentional control processes following the induction of positive mood in our study. Consistent with previous research (Ashby et al., 1999), we surmised that this change in positive mood following the MIP may be associated with a sustained increase in dopaminergic levels in specific cortical and subcortical structures related to executive functions. Nonetheless, it remains unclear at this stage whether the elected positive MIP actually yielded either phasic or tonic changes of dopamine levels in targeted prefrontal regions.

Previous studies have already shown that changes in positive mood are related to changes in cognitive functions, including the use of a more open (Estrada et al., 1997), creative (Isen et al., 1987), integrative (Isen et al., 1991), or flexible (Isen \& Daubman, 1984) thinking style. Taken together, these results point to a role of positive affective states in being able to trigger a broadening of the attentional scope (Derryberry \& Reed, 1994). On the basis of this evidence, Fredrickson and colleagues (Fredrickson, 2001; Fredrickson \& Levenson, 1998) advocated, in the broadenand-build theory, that negative mood would prompt a narrowing of thought-action tendencies and attentional focus, while positive mood would, on the contrary, broaden people's thought-action repertoires and attentional scope. This way, mood provides human organisms with an adaptive and flexible mechanism enabling them to efficiently cope with changing environmental demands, by dynamically modulating the way incoming information is processed and eventually stored in memory systems (Gray, 2004).

However, the evidence showing that positive affect can trigger a genuine broadening of visual attentional scope and induce a more global information-processing style is scarce. Previous studies had mainly used cognitive control or interference tasks, such as the flanker task (Rowe et al., 2007). Rowe et al. found that for participants in a positive mood, flankers had a greater interference effect on central taskrelevant stimuli than was the case in a neutral or a sad mood, even when the spacing between target and flankers was increased. This broadening effect seems to be related to changes in extrastriate visual cortex, at the level of the P1 (Moriya \& Nittono, 2011). These results showed an increased proneness to distraction and are consistent with broadened attention in a positive mood (Fredrickson, 2001; Fredrickson \& Levenson, 1998), even though a direct link between positive mood and changes in attention control mechanisms remains difficult to establish from these studies. Because these interference tasks primarily rely on several cognitive processes beyond attention (e.g., executive functions and cognitive control; see Botvinick, Braver, Barch, Carter, \& Cohen, 2001), it is unclear whether positive mood can causally lead to a change in the attention focus and in turn gate early sensory stimulus processing. Therefore, the main goal of our study was to address this question using a standard visuospatial task (Rauss et al., 2009; Rauss et al., 2011; Rossi \& Pourtois, 2012; Schwartz et al., 2005), capitalizing on the high temporal resolution provided by ERPs to track attention-dependent changes in early sensory processing triggered by the earlier induction of a positive mood.

Neurophysiological mechanism underlying broadening of attention

Participants were asked to perform a demanding oddball detection task at fixation, ensuring that voluntary attention was properly locked to this position in the visual field, but leaving enough attentional resources available for the covert processing of visual distractors (EEG experiment) or overt processing of exogenous task-relevant visual textures (behavioral experiment). These uniform visual textures were briefly flashed in the upper visual field at unpredictable times and variable locations. Using ERP measurements, we could thus track electrical brain activity unambiguously elicited by either the central or the peripheral stimuli and assess at which stage their respective sensory processing was modulated by the induction of positive mood. We reasoned that if positive mood truly broadens attention scope, then the sensory processing of the unattended peripheral distractors should be altered, especially for the unattended stimuli shown at the most extreme spatial location relative to fixation.

Our new ERP results confirm this conjecture and show that the earliest stage of stimulus processing in $\mathrm{V} 1$, as indexed by the $\mathrm{C} 1$, is influenced by the location of the distractor in the upper visual field, as well as by mood. The amplitude and polarity of the $\mathrm{C} 1$ systematically vary with the position of the stimulus in the visual field, reflecting an early retinotopic-encoding effect (Clark et al., 1995). Using a standard ERP topographic-mapping analysis (Pourtois et al., 2008), we found that in a neutral mood, participants showed a nonlinear reduction of the topographical $\mathrm{C} 1$ component with increasing distance between the distractor and fixation, reflecting the spatial sensitivity of the C1 (Clark et al., 1995). However, participants in the positive mood group did not show such a spatial-gradient effect. Hence, the spatial gradient of the $\mathrm{C} 1$ found in the neutral mood group was blurred after the induction of positive mood, showing a drop in selectivity for the early spatial encoding of distractors in V1. These findings show an interaction effect between bottom-up sensory processingguided by low-level stimulus information (Egeth \& Yantis, 
1997; Wolfe \& Horowitz, 2004), in this case the spatial location of the distractor stimulus shown in the upper visual field (Rauss et al., 2011) — and top-down factors (Buschman \& Miller, 2007; Corbetta \& Shulman, 2002; Marois \& Ivanoff, 2005; Theeuwes, 2010) such as the current mood state of the participant (Gray, 2004; Rossi \& Pourtois, 2012).

Note that this effect of mood on early sensory processing of the distractors was task-specific, mood-dependent, and selective for the $\mathrm{C} 1$ component in our study. During the localizer run, we found that the $\mathrm{C} 1$ component to these same peripheral distractors was not influenced by mood, although the electrophysiological properties of the $\mathrm{C} 1$ were very similar in the main attention task and the localizer (see Fig. 6). This might be explained by mood effects fading out during the recording of the localizer blocks and/or the task differences between the localizer (i.e., passive viewing conditions) and the main task blocks. In this sense, the absence of mood effects on the localizer blocks might indicate a specific effect of positive mood on active filtering of irrelevant information, instead of an unspecific influence of this factor on the mere early bottomup processing of visual stimuli in V1. However, future studies will be needed to assess and better characterize the (shortscale) time-dependent fluctuations of effects of (positive) mood, and how they may influence early visual and attention brain processes (including the $\mathrm{C} 1$ component). More specifically, whether positive mood selectively alters either spatial properties or feature-based components of (selective) attention (or both) will require additional empirical validation. Likewise, given the existing evidence showing the opposite effects of positive versus negative mood on global versus local information-processing styles (Gable \& Harmon-Jones, 2008, 2010; Gasper \& Clore, 2002; Rowe et al., 2007), additional work will be needed as well to assess whether positive mood primarily influences the information-processing style overall, or instead is best characterized by process-specific changes concerning attention control mechanisms.

Unlike the $\mathrm{C} 1$ component, analyses of the extrastriate $\mathrm{P} 1$ component (Hillyard \& Anllo-Vento, 1998; Martínez et al., 1999) failed to reveal any change as a function of stimulus position in the upper visual field, consistent with previous ERP findings (Clark et al., 1995). Likewise, mood had no effect on the topographical properties of the P1 component. However, given the strong anatomical connections between the prefrontal cortex and the parietal and occipital cortices, a modulatory effect of positive mood remotely influencing not only early visual areas (including V1) but also the extrastriate visual cortex (and, hence, the P1 component) appears more likely. Moreover, our observation of a $\mathrm{C} 1$-selective effect of positive mood could also be explained by the specific task demands and stimulus parameters used in our study. The experimental paradigm that we elected likely promoted attention competition/selection primarily in the spatial domain (see also Rossi \& Pourtois, 2012).We surmise that a modulation of the P1 component by (positive) mood could also have been found if different task demands and stimulus characteristics had been used-for example, dot probe or cueing tasks (see Pourtois, Grandjean, Sander, \& Vuilleumier, 2004).

Another important finding of our study was to show that in both experiments, mood did not change the processing of the centrally presented/task-relevant stimuli. As expected, the amplitude of the P300 component was strongly influenced by task demands (Kim et al., 2008; Kok, 2001; McCarthy \& Donchin, 1981; Sawaki \& Katayama, 2007), being larger for perceived deviant than for standard targets. However, this effect was not different between the two mood groups, in agreement with earlier ERP studies (Moriya \& Nittono, 2011; Rossi \& Pourtois, 2012). Moriya and Nittono used a flanker task, but they did not report any reliable effect of either positive or negative mood on the amplitude of the P300 component. They concluded that attention might only be influenced by mood at early stages of stimulus processing, as opposed to response selection and decision processes. Rossi and Pourtois (2012) also confirmed that neither positive nor negative mood influenced the amplitude of the target-related P300 component (as well as accuracy at the behavioral level), using an adapted version of the experimental paradigm used in this study. In their ERP study, participants were instructed to attend to a RSVP stream at fixation, consisting of the same line segments as in the present study. In some trials, a deviant line orientation was presented, and these deviant lines had to be detected by participants (ratio of standard: target line orientations was 4:1). The perceptual load for these central stimuli was manipulated in their study, such that the detection task could be easy, intermediate, or difficult. Participants performed this task under either a positive or a negative affective state (within-subjects design). Their results showed that the target-related P300 component, as well as accuracy at the behavioral level, varied strongly and in a predictive direction depending on task difficulty. However, and importantly, mood did not interact with these effects for the easiest or the most difficult level of task difficulty. By contrast (and similarly to the present study), in this earlier study mood altered the early visual processing of (unattended) peripheral distractors, suggesting that (statedependent) affect mainly influenced peripheral (and unattended) stimuli, as opposed to central vision (and the processing of attended stimuli). The new ERP results presented in our study (see Exp. 1) are compatible with this interpretation, and they confirmed an asymmetry between central/ attended and peripheral/unattended locations for effects of mood or affect on (early) visual perception. In contrast, in another study the researchers did find a modulation of the P300 component by negative affective state (threat of shock; 
Shackman et al., 2011). Hence, it remains unclear whether the decision-related P300 component can be modulated by affective state. More generally, our results suggest that positive mood may lead to qualitatively different effects during sensory processing and decision-making processes for taskrelevant stimuli, relative to negative emotions. Future ERP studies that more directly compare the effects of positive versus negative mood will be needed to assess whether not only early sensory-processing stages (e.g., the $\mathrm{C} 1$ component), but also later decision-related processes are equally influenced by these opposite affective states. Likewise, it appears necessary to explore further the likely dependency of some of these ERP components on specific neurotransmitter systems. In particular, given that positive mood is thought to be mediated by specific dopaminergic systems (Ashby et al., 1999), some of the present ERP effects could tentatively be related to changes in these dopaminergic systems. In this regard, studies linking the P300 component either to dopaminergic (see Pogarell et al., 2011) or adrenergic (Nieuwenhuis, Aston-Jones, \& Cohen, 2005; Polich, 2007) inputs would provide important hints on the actual organization of the underlying brain architecture and neural systems mediating the effects of (positive) mood on attention.

More generally, our findings suggest that the effects of positive mood were specific to the processing of peripheral, task-irrelevant stimuli and that they did not influence taskrelevant, central stimuli. This result allows us to rule out the possibility that changes in early sensory processing at the level of the $\mathrm{C} 1$ for the peripheral visual textures could be explained by systematic behavioral performance imbalances between mood groups for the task at fixation. The behavioral data collected during the EEG experiment as well as during the additional behavioral experiment, showing no difference in accuracy or RTs between mood groups for the task at fixation, further support this conclusion.

Additional information regarding the nature and extent of the selective change produced by positive mood was provided by the additional behavioral experiment. We reasoned that if the effect of positive mood may correspond to a drop in early spatial-encoding selectivity, as our new ERP results for the $\mathrm{C} 1$ suggest, the capacity to discriminate subtle geometric differences between different peripheral stimuli may be impaired in positive mood, as compared to a neutral mood, given the intrinsic attention competition exerted by the three nonoverlapping spatial positions in the upper visual field. This new result is compatible with earlier findings in the literature, showing that a larger attentional scope may lead to an associated loss in processing efficiency and spatial resolution (as compared to a smaller scope; Castiello \& Umiltà, 1990, 1992; Eriksen \& Yeh, 1985) and that this effect is reflected in a decreased neural signal change in the corresponding retinotopic area (Müller et al.,
2003).These results provide support for the assumption of a trade-off effect between the size of the attentional focus and the efficiency/resolution of visual (spatial) processing (Castiello \& Umiltà, 1990, 1992; Eriksen \& Yeh, 1985; Ivry \& Robertson, 1998; Müller et al., 2003). In line with this reasoning, when peripheral textures became task-relevant and had to be discriminated (Exp. 2), accuracy dropped as a function of the distance of the textures relative to fixation. Similarly to these previous findings showing a drop in the efficiency of stimulus processing when attentional scope was broadened, we found that participants in the positive mood group committed on average substantially more discrimination errors than did participants in the neutral mood group across the three positions. When combined with our new $\mathrm{C} 1$ results showing altered sensitivity between the three positions in the upper visual field in a positive relative to a neutral mood (Exp. 1), this decrease in accuracy in the positive mood group might suggest a drop in attention selectivity across these three positions in the upper visual field (Exp. 2). However, some caution is needed when directly comparing the results of these experiments, given that they differed along several dimensions. While working memory was presumably taxed similarly in both experiments by the central RSVP, in Experiment 2 the peripheral textures were also task-relevant and required an explicit visual discrimination, possibly triggering a more open attention focus, as compared to the task-irrelevant distractors in Experiment 1. Moreover, if we assume a general, limitedresources account of attention capacities (see Marois \& Ivanoff, 2005), then increasing task demands may block or dampen the effects of (positive) mood on early visual perception. Nonetheless, in Experiment 1, we found clear evidence for an effect of the position of textures in the upper peripheral visual field at the level of the $\mathrm{C}$, indicative of a change in the distribution of spatial attention in this portion of the visual field, as well as a significant modulation of this effect by a positive mood. Future studies will be needed to assess whether the task relevance of peripheral textures reliably influences the size and extent of positive mood effects (on early visual perception). The results of our study (Exp. 2) showed that despite a dual-task setting and a (high) working memory load, positive mood could still exert a modulatory effect on the ability to discriminate peripheral textures shown in the upper visual field, an effect that presumably arises early following stimulus onset in the primary visual cortex when the locations of these textures were precisely/retinotopically encoded (see the results of Exp. 1).

Presumably, if positive emotion broadens attention, the distractor or location specificity may be impaired, because attention is by definition operating over a potentially more expanded region. Changes in dopaminergic-dependent prefrontal attention control mechanisms under positive mood 
could underlie these early visual perception effects (Ashby et al., 1999; Lavie, 1995). However, the actual mechanisms linking positive mood, dopamine, and cognitive processes are not yet fully understood, partly due to the existence of several dopamine receptor types and different dopaminergic-dependent neuromodulation pathways, connecting to different (sub)cortical brain regions. It is likely that the influence of sustained positive mood (as achieved in this study) on specific cognitive processes, such as attention, actually concerns tonic changes in dopamine levels, as opposed to mere phasic fluctuations. Alternatively, it has been suggested that phasic dopamine increases in prefrontal cortex, elicited by stimuli that are rewarding or reward-predicting, serve as a pervasive gating signal. Accordingly, we surmise that the positive MIP, instructing participants to focus on a personal experience that generated a positive mood, served as a reward cue for the participants, hence likely influencing phasic dopamine release in this region, even though future studies will be needed to corroborate this conclusion.

\section{Broadening through decreased inhibition?}

The alteration in early sensory processing of distractors under positive mood (and the behavioral effect of this alteration) might be explained by a change in higherlevel attention control mechanisms (Corbetta \& Shulman, 2002; Lavie, 2005). It is possible that resources left over and not consumed by the main oddball task at fixation may be used to process covertly these peripheral stimuli (Lavie, 1995, 2005; Lavie et al., 2004; Lavie \& Tsal, 1994), and this effect could be exacerbated under positive mood. Hence, positive mood may not influence sensory processing in V1 directly, but rather (tonically) loosen the normal top-down control exerted by prefrontal attention control regions onto V1 (see also Rossi \& Pourtois, 2012). Therefore, the effect of positive mood in our study might very well operate at this level and interfere with the normal recruitment of prefrontal cognitive control regions aimed at downplaying the distraction effect induced by these peripheral visual textures. These prefrontal regions primarily include the dorsolateral prefrontal cortex (Miller \& Cohen, 2001; Posner \& Presti, 1987), as well as the anterior cingulate cortex (Posner \& Petersen, 1990; Ridderinkhof, Ullsperger, Crone, \& Nieuwenhuis, 2004). Interestingly, because both regions are receiving strong dopaminergic inputs from the midbrain and basal ganglia, and because positive mood may be associated with a tonic change in these dopaminergic brain systems (Ashby et al., 1999), the observed changes in V1 after the induction of positive mood in our study may tentatively be linked to these distant prefrontal effects, or alternatively, to a more global change in the frontoparietal network supporting the endogenous control of attention (Corbetta \& Shulman, 2002). In other words, positive mood might affect these prefrontal inhibitory systems (Wang et al., 2011), eventually leading to a change in early sensory processing in V1, given the strong anatomical projections from these prefrontal areas to early sensory cortices, including V1 (Van Essen, Anderson, \& Felleman, 1992). Hence, because of this decreased prefrontal control or inhibitory mechanism, positive emotion alters early sensory processing in V1, and hence attention selectivity, revealing in turn a downside of positive emotion on early visual cognition (see also the results of the behavioral experiment, corroborating this conclusion). One may assume that a similar release in prefrontal inhibitory control may explain a variety of effects observed under positive mood, including a more global (as opposed to local) processing style (Gable \& Harmon-Jones, 2010), a higher susceptibility to distraction during interference tasks (Rowe et al., 2007), and lower adaptation following conflict detection (van Steenbergen et al., 2010), as well as, eventually, an enhanced creative style (Isen, 2000; Isen \& Daubman, 1984; Isen et al., 1987; Isen et al., 1991). However, it should be mentioned that the focus of our study was on how "low-level" attentional and visual processes could be modulated by (transient and shortlived) changes in levels of positive mood, as opposed to alterations of high-level cognitive functions, such as creativity, reasoning, problem solving, or language. Interestingly, it is plausible to assume that a common "basic" process might underlie changes seen in a broad range of cognitive functions after the induction of positive emotion. More specifically, a substantial decrease in frontal inhibitory processes following the induction of positive mood, such as has been postulated in earlier research or models (Biss \& Hasher, 2011; Biss et al., 2010; Rowe et al., 2007), might possibly explain, although indirectly, the present ERP results, as well as a wide range of behavioral phenomena previously reported in the literature, including the adoption of a more creative and flexible information processing style. Additional brain-imaging studies will be needed, however, to more directly link changes in such prefrontal inhibitory control mechanisms with the induction of positive mood, and finally to try to causally relate these putative changes in higher prefrontal brain regions with specific alterations during early sensory processing or attention control, as revealed in our study.

Author note R.D.R. and G.P. are funded by a Concerted Research Action Grant from Ghent University (BOF10/GOA/014). G.P. is funded by the European Research Council (Starting Grant No. 
200758) and by Ghent University (BOF Grant No. 05Z01708).We thank the three reviewers for their comments on an earlier version of the manuscript.

\section{References}

Ashby, F. G., Isen, A. M., \& Turken, A. U. (1999). A neuropsychological theory of positive affect and its influence on cognition. Psychological Review, 106, 529-550. doi:10.1037/0033295X.106.3.529

Beck, A. T., Steer, R. A., Ball, R., \& Ranieri, W. F. (1996). Comparison of Beck Depression Inventories-IA and -II in psychiatric outpatients. Journal of Personality Assessment, 67, 588-597. doi:10.1207/s15327752jpa6703_13

Biss, R. K., \& Hasher, L. (2011). Delighted and distracted: Positive affect increases priming for irrelevant information. Emotion, 11, 1474-1478.

Biss, R. K., Hasher, L., \& Thomas, R. C. (2010). Positive mood is associated with the implicit use of distraction. Motivation and Emotion, 34, 73-77.

Botvinick, M. M., Braver, T. S., Barch, D. M., Carter, C. S., \& Cohen, J. D. (2001). Conflict monitoring and cognitive control. Psychological Review, 108, 624-652. doi:10.1037/0033295X.108.3.624

Bower, G. H., \& Mayer, J. D. (1989). In search of mood-dependent retrieval. Journal of Social Behavior and Personality, 4, 121-156.

Buschman, T. J., \& Miller, E. K. (2007). Top-down versus bottom-up control of attention in the prefrontal and posterior parietal cortices. Science, 315, 1860-1862. doi:10.1126/science.1138071

Carrasco, M. (2011). Visual attention: The past 25 years. Vision Research, 51, 1484-1525. doi:10.1016/j.visres.2011.04.012

Carver, C. S., \& White, T. L. (1994). Behavioral-inhibition, behavioral activation, and affective responses to impending reward and punishment: The Bis-Bas scales. Journal of Personality and Social Psychology, 67, 319-333.

Castiello, U., \& Umiltà, C. (1990). Size of the attentional focus and efficiency of processing. Acta Psychologica, 73, 195-209.

Castiello, U., \& Umiltà, C. (1992). Splitting focal attention. Journal of Experimental Psychology: Human Perception and Performance, 18, 837-848. doi:10.1037/0096-1523.18.3.837

Clark, V. P., Fan, S., \& Hillyard, S. A. (1995). Identification of early visual evoked potential generators by retinotopic and topographic analyses. Human Brain Mapping, 2, 170-187.

Corbetta, M., \& Shulman, G. L. (2002). Control of goal-directed and stimulus-driven attention in the brain. Nature Reviews Neuroscience, 3, 201-215.

Crawford, J. R., \& Henry, J. D. (2004). The positive and negative affect schedule (PANAS): Construct validity, measurement properties and normative data in a large non-clinical sample. British Journal of Clinical Psychology, 43, 245-265.

Derryberry, D., \& Reed, M. A. (1994). Temperament and attention: Orienting toward and away from positive and negative signals. Journal of Personality and Social Psychology, 66, 1128-1139.

Desimone, R., \& Duncan, J. (1995). Neural mechanisms of selective visual attention. Annual Review of Neuroscience, 18, 193-222. doi:10.1146/annurev.ne.18.030195.001205

Desseilles, M., Balteau, E., Sterpenich, V., Dang-Vu, T. T., Darsaud, A., Vandewalle, G., ... Schwartz, S. (2009). Abnormal neural filtering of irrelevant visual information in depression. Journal of Neuroscience, 29, 1395-1403. doi:10.1523/ JNEUROSCI.3341-08.2009

Di Russo, F., Martínez, A., Sereno, M. I., Pitzalis, S., \& Hillyard, S. A. (2002). Cortical sources of the early components of the visual evoked potential. Human Brain Mapping, 15, 95-111.
Dreisbach, G., \& Goschke, T. (2004). How positive affect modulates cognitive control: Reduced perseveration at the cost of increased distractibility. Journal of Experimental Psychology: Learning, Memory, and Cognition, 30, 343-353. doi:10.1037/0278-7393.30.2.343

Egeth, H. E., \& Yantis, S. (1997). Visual attention: Control, representation, and time course. Annual Review of Psychology, 48, 269 297. doi:10.1146/annurev.psych.48.1.269

Ericsson, E., Olofsson, J. K., Nordin, S., Rudolfsson, T., \& Sandstrom, G. (2008). Is the P600/SPS affected by the richness of semantic content? A linguistic ERP study in Swedish. Scandinavian Journal of Psychology, 49, 1-9.

Eriksen, C. W., \& Yeh, Y. Y. (1985). Allocation of attention in the visual field. Journal of Experimental Psychology: Human Perception and Performance, 11, 583-597. doi:10.1037/0096-1523.11.5.583

Estrada, C. A., Isen, A. M., \& Young, M. J. (1997). Positive affect facilitates integration of information and decreases anchoring in reasoning among physicians. Organizational Behavior and Human Decision Processes, 72, 117-135.

Fredrickson, B. (2001). The role of positive emotions in positive psychology: The broaden-and-build theory of positive emotions. American Psychologist, 56, 218-226.

Fredrickson, B., \& Levenson, R. W. (1998). Positive emotions speed recovery from the cardiovascular sequelae of negative emotions. Cognition and Emotion, 12, 191-220.

Gable, P. A., \& Harmon-Jones, E. (2008). Approach-motivated positive affect reduces breadth of attention. Psychological Science, 19, 476-482. doi:10.1111/j.1467-9280.2008.02112.x

Gable, P. A., \& Harmon-Jones, E. (2010). The blues broaden, but the nasty narrows: Attentional consequences of negative affects low and high in motivational intensity. Psychological Science, 21, 211-215.

Gasper, K., \& Clore, G. L. (2002). Attending to the big picture: Mood and global versus local processing of visual information. Psychological Science, 13, 34-40. doi:10.1111/1467-9280.00406

Gomez Gonzalez, C. M., Clark, V. P., Fan, S., Luck, S. J., \& Hillyard, S. A. (1994). Sources of attention-sensitive visual event-related potentials. Brain Topography, 7, 41-51.

Gratton, G., Coles, M. G. H., \& Donchin, E. (1983). Filtering for spatialdistribution-A new approach (vector filter). Psychophysiology, 20, 443-444.

Gray, J. R. (2001). Emotional modulation of cognitive control: Approach-withdrawal states double-dissociate spatial from verbal two-back task performance. Journal of Experimental Psychology: General, 130, 436-452.

Gray, J. R. (2004). Integration of emotion and cognitive control. Current Directions in Psychological Science, 13, 46-48.

Herrmann, C. S., \& Knight, R. T. (2000). Mechanisms of human attention: Event-related potentials and oscillations. Neuroscience and Biobehavioral Reviews, 25, 465-476.

Hickey, C., Chelazzi, L., \& Theeuwes, J. (2010). Reward changes salience in human vision via the anterior cingulate. Journal of Neuroscience, 30, 11096-11103. doi:10.1523/JNEUROSCI.1026-10.2010

Hillyard, S. A., \& Anllo-Vento, L. (1998). Event-related brain potentials in the study of visual selective attention. Proceedings of the National Academy of Sciences, 95, 781-787.

Holmes, E. A. (2006). Positive interpretation training: Effects of mental imagery versus verbal training on positive mood. Behavior Therapy, 37, 237.

Holmes, E. A., Coughtrey, A. E., \& Connor, A. (2008). Looking at or through rose-tinted glasses? imagery perspective and positive mood. Emotion, 8, 875-879.

Isen, A. M. (2000). Positive affect and decision making. In M. Lewis \& J. M. Haviland-Jones (Eds.), Handbook of emotions (2nd ed., pp. 417-435). New York, NY: Guilford Press.

Isen, A. M., \& Daubman, K. A. (1984). The influence of affect on categorization. Journal of Personality and Social Psychology, 47, 1206-1217. 
Isen, A. M., Daubman, K. A., \& Nowicki, G. P. (1987). Positive affect facilitates creative problem-solving. Journal of Personality and Social Psychology, 52, 1122-1131.

Isen, A. M., Rosenzweig, A. S., \& Young, M. J. (1991). The influence of positive affect on clinical problem-solving. Medical Decision Making, 11, 221-227.

Ivry, R. B., \& Robertson, L. C. (1998). The two sides of perception. Cambridge, MA: MIT Press.

Jeffreys, D. A., \& Axford, J. G. (1972). Source locations of patternspecific components of human visual evoked-potentials: 1 . Component of striate cortical origin. Experimental Brain Research, 16, 1-21.

Kim, K. H., Kim, J. H., Yoon, J., \& Jung, K.-Y. (2008). Influence of task difficulty on the features of event-related potential during visual oddball task. Neuroscience Letters, 445, 179-183.

Kok, A. (2001). On the utility of P3 amplitude as a measure of processing capacity. Psychophysiology, 38, 557-577.

Kringelbach, M. L., \& Rolls, E. T. (2004). The functional neuroanatomy of the human orbitofrontal cortex: Evidence from neuroimaging and neuropsychology. Progress in Neurobiology, 72, 341-372.

Lavie, N. (1995). Perceptual load as a necessary condition for selective attention. Journal of Experimental Psychology: Human Perception and Performance, 21, 451-468. doi:10.1037/00961523.21.3.451

Lavie, N. (2005). Distracted and confused? Selective attention under load. Trends in Cognitive Sciences, 9, 75-82. doi:10.1016/ j.tics.2004.12.004

Lavie, N., Hirst, A., de Fockert, J. W., \& Viding, E. (2004). Load theory of selective attention and cognitive control. Journal of Experimental Psychology: General, 133, 339-354. doi:10.1037/ 0096-3445.133.3.339

Lavie, N., \& Tsal, Y. (1994). Perceptual load as a major determinant of the locus of selection in visual attention. Perception \& Psychophysics, 56, 183-197. doi:10.3758/BF03213897

Marois, R., \& Ivanoff, J. (2005). Capacity limits of information processing in the brain. Trends in Cognitive Sciences, 9, 296-305. doi:10.1016/j.tics.2005.04.010

Martínez, A., Anllo-Vento, L., Sereno, M.I., Frank, L.R., Buxton, R. B., Dubowitz, D.J., ... Hillyard, S.A. (1999). Involvement of striate and extrastriate visual cortical areas in spatial attention. Nature Neuroscience, 2, 364-369. doi:10.1038/7274

McCarthy, G., \& Donchin, E. (1981). A metric for thought: A comparison of P300 latency and reaction time. Science, 211, 77-80.

Michel, C. M., Seeck, M., \& Landis, T. (1999). Spatiotemporal dynamics of human cognition. News in Physiological Sciences, 14, 206-214.

Miller, E. K., \& Cohen, J. D. (2001). An integrative theory of prefrontal cortex function. Annual Review of Neuroscience, 24, 167-202. doi:10.1146/annurev.neuro.24.1.167

Mitterschiffthaler, M. T., Fu, C. H. Y., Dalton, J. A., Andrew, C. M., \& Williams, S. C. R. (2007). A functional MRI study of happy and sad affective states induced by classical music. Human Brain Mapping, 28, 1150-1162.

Moriya, H., \& Nittono, H. (2011). Effect of mood states on the breadth of spatial attentional focus: An event-related potential study. Neuropsychologia, 49, 1162-1170. doi:10.1016/ j.neuropsychologia.2011.02.036

Müller, N. G., Bartelt, O. A., Donner, T. H., Villringer, A., \& Brandt, S. A. (2003). A physiological correlate of the "Zoom Lens" of visual attention. Journal of Neuroscience, 23, 3561-3565.

Murray, M. M., Brunet, D., \& Michel, C. M. (2008). Topographic ERP analyses: A step-by-step tutorial review. Brain Topography, 20, 249-264.

Nieuwenhuis, S., Aston-Jones, G., \& Cohen, J. D. (2005). Decision making, the P3, and the locus coeruleus-norepinephrine system. Psychological Bulletin, 131, 510-532. doi:10.1037/00332909.131.4.510
Pascual-Marqui, R. D., Michel, C. M., \& Lehmann, D. (1995). Segmentation of brain electrical activity into microstates: Model estimation and validation. IEEE Transactions in Biomedical Engineering, 42, 658-665.

Pessoa, L., \& Engelmann, J. B. (2010). Embedding reward signals into perception and cognition. Frontiers in Neuroscience, 4, 1-8.

Pogarell, O., Padberg, F., Karch, S., Segmiller, F., Juckel, G., Mulert, C., ... Koch, W. (2011). Dopaminergic mechanisms of target detection-P300 event related potential and striatal dopamine. Psychiatry Research: Neuroimaging, 194, 212-218. doi:10.1016/j.pscychresns.2011.02.002

Polich, J. (2007). Updating p300: An integrative theory of P3a and P3b. Clinical Neurophysiology, 118, 2128-2148.

Polich, J., \& Kok, A. (1995). Cognitive and biological determinants of P300: An integrative review. Biological Psychology, 41, 103-146.

Posner, M. I., \& Petersen, S. E. (1990). The attention system of the human brain. Annual Review of Neuroscience, 13, 25-42.

Posner, M. I., \& Presti, D. E. (1987). Selective attention and cognitive control. Trends in Neurosciences, 10, 13-17.

Posner, M. I., Snyder, C. R., \& Davidson, B. J. (1980). Attention and the detection of signals. Journal of Experimental Psychology: General, 109, 160-174. doi:10.1037/0096-3445.109.2.160

Pourtois, G., Dan, E. S., Grandjean, D., Sander, D., \& Vuilleumier, P. (2005a). Enhanced extrastriate visual response to bandpass spatial frequency filtered fearful faces: Time course and topographic evoked-potentials mapping. Human Brain Mapping, 26, 65-79. doi:10.1002/hbm.20130

Pourtois, G., Delplanque, S., Michel, C., \& Vuilleumier, P. (2008). Beyond conventional event-related brain potential (ERP): Exploring the time-course of visual emotion processing using topographic and principal component analyses. Brain Topography, 20, 265-277. doi:10.1007/s10548-0080053-6

Pourtois, G., De Pretto, M., Hauert, C. A., \& Vuilleumier, P. (2006). Time course of brain activity during change blindness and change awareness: Performance is predicted by neural events before change onset. Journal of Cognitive Neuroscience, 18, 21082129. doi:10.1162/jocn.2006.18.12.2108

Pourtois, G., Grandjean, D., Sander, D., \& Vuilleumier, P. (2004). Electrophysiological correlates of rapid spatial orienting towards fearful faces. Cerebral Cortex, 14, 619-633. doi:10.1093/cercor/ bhh023

Pourtois, G., Thut, G., Grave de Peralta, R., Michel, C., \& Vuilleumier, P. (2005b). Two electrophysiological stages of spatial orienting towards fearful faces: Early temporo-parietal activation preceding gain control in extrastriate visual cortex. NeuroImage, 26, 149163. doi:10.1016/j.neuroimage.2005.01.015

Rauss, K. S., Pourtois, G., Vuilleumier, P., \& Schwartz, S. (2009). Attentional load modifies early activity in human primary visual cortex. Human Brain Mapping, 30, 1723-1733. doi:10.1002/ hbm. 20636

Rauss, K. S., Schwartz, S., \& Pourtois, G. (2011). Top-down effects on early visual processing in humans: A predictive coding framework. Neuroscience and Biobehavioral Reviews, $35,1237-1253$

Reisberg, D., Pearson, D. G., \& Kosslyn, S. M. (2003). Intuitions and introspections about imagery: The role of imagery experience in shaping an investigator's theoretical views. Applied Cognitive Psychology, 17, 147-160.

Ridderinkhof, K. R., Ullsperger, M., Crone, E. A., \& Nieuwenhuis, S. (2004). The role of the medial frontal cortex in cognitive control. Science, 306, 443-447. doi:10.1126/science.1100301

Rolls, E. T. (2000). The orbitofrontal cortex and reward. Cerebral Cortex, 10, 284-294.

Rossi, V., \& Pourtois, G. (2011). Transient state-dependent fluctuations in anxiety measured using STAI, POMS, PANAS or VAS: A comparative review. Anxiety, Stress, and Coping, 1-43. 
Rossi, V., \& Pourtois, G. (2012). State-dependent attention modulation of human primary visual cortex: A high density ERP study. NeuroImage, 60, 2365-2378.

Rowe, G., Hirsh, J. B., \& Anderson, A. K. (2007). Positive affect increases the breadth of attentional selection. Proceedings of the National Academy of Sciences, 104, 383-388.

Russell, J. A., \& Carroll, J. M. (1999). On the bipolarity of positive and negative affect. Psychological Bulletin, 125, 3-30.

Sawaki, R., \& Katayama, J. (2007). Difficulty of discrimination modulates attentional capture for deviant information. Psychophysiology, 44, 374-382.

Schwartz, S., Vuillemier, P., Hutton, C., Maravita, A., Dolan, R. J., \& Driver, J. (2005). Attentional load and sensory competition in human vision: Modulation of fMRI responses by load at fixation during task-irrelevant stimulation in the peripheral visual field. Cerebral Cortex, 15, 770-786.

Shackman, A. J., Maxwell, J. S., McMenamin, B. W., Greischar, L. L., \& Davidson, R. J. (2011). Stress potentiates early and attenuates late stages of visual processing. Journal of Neuroscience, 31, 1156-1161.

Spielberger, C. D., Gorsuch, R. L., Lushene, R., Vagg, P. R., \& Jacobs, G. A. (1983). Manual for the State-Trait Anxiety Inventory. Palo Alto, CA: Consulting Psychologists Press.

Stolarova, M., Keil, A., \& Moratti, S. (2006). Modulation of the C1 visual event-related component by conditioned stimuli: Evidence for sensory plasticity in early affective perception. Cerebral Cortex, 16, 876-887.

Theeuwes, J. (2010). Top-down and bottom-up control of visual selection. Acta Psychologica, 135, 77-99. doi:10.1016/j.actpsy.2010.02.006

Tibshirani, R., Walther, G., \& Hastie, T. (2001). Estimating the number of clusters in a data set via the gap statistic. Journal of the Royal Statistical Society Series B, 63, 411-423.
Van Essen, D. C., Anderson, C. H., \& Felleman, D. J. (1992). Information processing in the primate visual system: An integrated systems perspective. Science, 255, 419-423.

van Steenbergen, H., Band, G. P. H., \& Hommel, B. (2009). Reward counteracts conflict adaptation: Evidence for a role of affect in executive control. Psychological Science, 20, 1473-1477. doi:10.1111/j.1467-9280.2009.02470.x

van Steenbergen, H., Band, G. P. H., \& Hommel, B. (2010). In the mood for adaptation: How affect regulates conflict-driven control. Psychological Science, 21, 1629-1634.

Wang, Y., Yang, J. M., Yuan, J. J., Fu, A. G., Meng, X. X., \& Li, H. (2011). The impact of emotion valence on brain processing of behavioral inhibitory control: Spatiotemporal dynamics. Neuroscience Letters, 502, 112-116.

Watkins, E. R., \& Moberly, N. J. (2009). Concreteness training reduces dysphoria: A pilot proof-of-principle study. Behaviour Research and Therapy, 47, 48-53.

Watson, D., Clark, L. A., \& Tellegen, A. (1988). Development and validation of brief measures of positive and negative affect: The PANAS scales. Journal of Personality and Social Psychology, 54, 1063-1070. doi:10.1037/0022-3514.54.6.1063

Watson, D., Wiese, D., Vaidya, J., \& Tellegen, A. (1999). The two general activation systems of affect: Structural findings, evolutionary considerations, and psychobiological evidence. Journal of Personality and Social Psychology, 76, 820-838.

West, G. L., Anderson, A. A., Ferber, S., \& Pratt, J. (2011). Electrophysiological evidence for biased competition in V1 for fear expressions. Journal of Cognitive Neuroscience, 23, 3410-3418.

Wolfe, J. M., \& Horowitz, T. S. (2004). What attributes guide the deployment of visual attention and how do they do it? Nature Reviews Neuroscience, 5, 495-501. doi:10.1038/nrn1411 